\title{
Cell-Cell Adhesion During Nephron Development Is Driven by Wnt/PCP Formin Daam1
}

Vanja Krneta-Stankic1,2, Mark Corkins2, Adriana Paulucci-Holthauzen3, Malgorzata Kloc3,4,

Andrew Gladden1,3,5, Rachel Miller1,2,3,6, *

6

${ }_{1}$ MD Anderson Cancer Center UTHealth Graduate School of Biomedical Sciences, Program in Genes and Development, Houston, TX, USA 2Department of Pediatrics, Pediatric Research Center, UTHealth McGovern Medical School, Houston, TX, USA ${ }_{3}$ Department of Genetics, University of Texas MD Anderson Cancer Center, Houston, TX, USA 4Houston Methodist Hospital Research Institute, Houston, TX, USA 5Department of Pathology and Laboratory Medicine, University of North Carolina, Chapel Hill, NC, USA

${ }_{6} \mathrm{MD}$ Anderson Cancer Center UTHealth Graduate School of Biomedical Sciences, Program in Biochemistry and Cell Biology, Houston, TX, USA

${ }^{\star}$ Correspondence: Rachel.K.Miller@uth.tmc.edu

\section{SUMMARY}

E-cadherin junctions facilitate the assembly and disassembly of cell-cell contacts that drive development and homeostasis of epithelial tissues. The stability of E-cadherin-based junctions highly depends on their attachment to the actin cytoskeleton, but little is known about how the assembly of junctional actin filaments is regulated. Formins are a conserved group of proteins responsible for the formation and elongation of filamentous actin (F-actin). In this study, using Xenopus embryonic kidney and Madin-Darby canine kidney (MDCK) cells, we investigate the role of the Wnt/ planar cell polarity (PCP) formin protein Daam1 (Dishevelled-associated activator of morphogenesis 1 ) in regulating E-cadherin based intercellular adhesion. Using live imaging we show that Daam1 localizes to newly formed cell-cell contacts in the developing nephron. Furthermore, analyses of junctional F-actin upon Daam1 depletion indicate a decrease in microfilament localization and their slowed turnover. We also show that Daam1 is necessary for efficient and timely localization of junctional E-cadherin, which is mediated by Daam1's formin homology domain 2 (FH2). Finally, we establish that Daam1 signaling is 
33 essential for promoting organized movement of renal cells. This study demonstrates that Daam1

34 formin junctional activity is critical for epithelial tissue organization.

\section{Keywords}

37 E-cadherin; F-actin; adhesion; kidney; nephron; Daam1; formin; Wnt; planar cell polarity;

38 Xenopus; collective cell movements; convergence and extension; tubulogenesis

\section{INTRODUCTION}

41 Extensive cellular rearrangements with changes in cell shape drive morphogenesis, including

42 for example, the process of tubulogenesis. To execute these processes successfully, cells must

43 be able to interact with each other and their environment in a timely and coordinated manner.

44 These interactions entail transduction of specific signals arising from adhesive contacts with the

45 extracellular matrix (ECM) and neighboring cells. How cells remodel their adhesions is one of

46 the central questions in epithelial tissue biology.

48 The cadherin family of cell adhesion proteins such as E-cadherin facilitate intercellular adhesion

49 and formation of cellular junctions (Adams et al., 1998; Takeichi, 2014; Yap et al., 2015).

50 Changes in E-cadherin-based adhesion are associated with developmental disorders and

51 progression of disease (Friedl and Mayor, 2017; Mendonsa et al., 2018). E-cadherin levels at

52 intercellular contacts depend on the organization of the actin cytoskeleton, but much remains to

53 be learned about how the actin assembly is regulated at these adhesive sites. Moreover, much

54 of our understanding of in vivo actin regulation and dynamics in cell-cell adhesion derives from

55 observations in cell culture systems, invertebrate embryos and the vertebrate skin.

56 Understanding of junction dynamics in intact vertebrae tissues is challenging, due to technical

57 limitations and tissue inaccessibility. In this study, we probe the role of the formin protein

58 Dishevelled-associated activator of morphogenesis 1 (Daam1) in intercellular adhesion during 
kidney development using Xenopus laevis embryonic kidney and Madin-Darby canine kidney

60 (MDCK) cells.

62 Similar to many organs in our body, the kidney consists of a network of epithelial tubules. The

63 epithelial tubules of the kidney are called nephrons whose morphology is vital to kidney function.

64 Mesenchymal-epithelial transitions (MET) and coordinated cell rearrangements facilitate

65 nephron morphogenesis. Nephrons arise from the mass of mesenchymal cells that undergo

66 MET to form tubules consisting of tightly connected epithelial cells (McMahon, 2016; Saxen,

67 1987). Oriented cell intercalations drive the elongation of nephric tubules through a process

68 called convergent-extension (CE) (Castelli et al., 2013; Karner et al., 2009; Kunimoto et al.,

69 2017; Lienkamp et al., 2012). Extensive cytoskeletal rearrangements characterized by changes

70 in cell shape and coordinated cell movements accompany CE. Although E-cadherin-based

71 adhesions are implicated in mediating both MET and maintenance of coordinated cell

72 rearrangements (Campbell and Casanova, 2016), very little is known about how they function in

73 nephrogenesis (Combes et al., 2015; Lefevre et al., 2017; Marciano et al., 2011; Vestweber et

74 al., 1985).

76 Daam1 is a formin protein required for nephric tubulogenesis (Miller et al., 2011). Formin

77 proteins coordinate the organization of the actin cytoskeleton by nucleating and polymerizing

78 unbranched actin filaments. While Rho GTPases activate most formins, the activation of Daam1

79 depends on its interaction with Dishevelled (Dvl), a key intracellular component of the Wnt

80 signaling pathway (Liu et al., 2008). The Wnt signaling pathway plays important roles in nephron

81 development (McMahon, 2016; Miller and McCrea, 2009). The secreted Wnt ligands bind

82 Frizzled $(\mathrm{Fz})$ receptors and subsequently, via Dvl regulate the canonical ( $\beta$--catenin-dependent)

83 and non-canonical ( $\beta$--catenin-independent)/planar cell polarity (PCP) signaling. While the

84 canonical signaling commonly governs inductive events and cell fate, the non-canonical/PCP 
85 branch is associated with influencing cell behaviors and morphology. Nonetheless, the roles for

86 different branches of the Wnt pathway continue to evolve as recent studies provide evidence for

87 cross-talk between these two branches (Nagy et al., 2016; O’Brien et al., 2018). Dvl regulates

88 the non-canonical/PCP branch of the Wnt pathway through direct interaction with Daam1 (Liu et 89 al., 2008).

90

91 Increasing evidence suggests that formins may function as key regulators of the actin assembly

92 at the cell-cell junctions (Grikscheit and Grosse, 2016). Recent work in a mouse mammary

93 gland epithelial cell line, for example, has indicated that Daam1 is important for the stability of

94 epithelial cell junctions (Nishimura et al., 2016). Here, we expand on these findings by

95 examining the functional role of Daam1 in cellular junctions in the context of tissue

96 morphogenesis by analyzing its role in nephron development. Furthermore, using live cell

97 imaging we show that during establishment of cellular junctions, Daam1 first localizes to cellular

98 protrusions that initiate cell-cell contact, and subsequently, to newly formed junctions to promote

99 their stability. We find that Daam1 facilitates nephron morphogenesis by regulating the

100 assembly of junctional filamentous actin (F-actin) and in turn promotes the E-cadherin-based

101 epithelial adhesion.

\section{RESULTS}

104 Daam1 co-localizes with F-actin and E-cadherin within the nephric primordium

105 Knockdown of Daam1 in Xenopus disrupts nephron morphology without apparent effect on the

106 expression of genes related to early differentiation events (Miller et al., 2011). Differentiation

107 signals driving development of the nephric mesoderm in Xenopus largely function before the 108 onset of tubular morphogenesis (Vize et al., 2003). To further probe the mechanism by which

109 Daam1 regulates the shaping of nephrons, we analyzed the subcellular localization of

110 fluorescently tagged Daam1 at the beginning of tubular morphogenesis (around NF stage 30). 
111 Through kidney-targeted microinjections that were targeted to early embryonic cells fated to

112 contributed to kidney (presumptive nephron progenitors) (DeLay et al., 2016; Moody and Kline,

113 1990), we expressed 1ng of fluorescently tagged Daam1 mRNA in the presumptive nephron

114 progenitors and analyzed its localization in fixed and live tissue.

116 For analyses of fixed tissue, embryos were subjected to whole-mount staining. The samples

117 were stained with an antibody against GFP to visualize Daam1, and an antibody against Lhx1 to

118 label nephron progenitors (DeLay et al., 2018; Venegas-FERRÍN et al., 2010). An additional

119 staining with Phalloidin allowed us to visualize the F-actin cytoskeleton, or alternatively, another

120 antibody was used to define the localization of E-cadherin (Figure 1). Consistent with its

121 previously reported subcellular localization in other cell types (Corkins et al., 2019; Higashi et

122 al., 2019; Jaiswal et al., 2013; Kawabata Galbraith et al., 2018; Kida et al., 2007; Nishimura et

123 al., 2012, 2016), Daam1 co-localizes with patches of F-actin, showing a more diffuse staining

124 pattern in the cytoplasm and a strong localization to cell junctions (Figure 1A). Furthermore,

125 co-immunostaining for GFP and E-cadherin demonstrated that E-cadherin is expressed within

126 the nephric primordium and it co-localizes with Daam1 at the cell-cell junctions (Figure 1B).

128 During the early stages of development, the opaqueness of the Xenopus epithelium hinders

129 imaging of fluorescent protein expression in internal tissues, including the pronephric

130 primordium. In fixed tissues, this is overcome by using clearing agents such as BA:BB (1:2

131 mixture of benzyl alcohol and benzyl benzoate, aka Murray's clear). However, this process

132 requires dehydration of samples in methanol or ethanol prior to clearing, which is incompatible

133 with the use of fluorescent phalloidins (Becker and Gard, 2006). To overcome this obstacle, we

134 briefly washed embryos (<20 sec) in isopropanol prior to clearing with BA:BA (Nworu et al.,

135 2014; Strickland et al., 2004). This allowed us to visualize Daam1 in conjunction with F-actin in

136 intact pronephric primordium (Figure 1A). 
138 To overcome an analogous challenge in live embryos, we developed a novel way of imaging the

139 nephric primordium in vivo (Figure 2A). Previous studies have used the "windowed" embryo

140 approach consisting of microsurgical removal of the surface ectoderm to expose and image

141 underlying tissue (Kim and Davidson, 2013). Adopting this approach, we created "kidney-

142 windowed" embryos by removing the surface epithelium and exposing the underlying nephric

143 primordium for high-resolution in vivo imaging. In vivo time-lapse imaging of "kidney-windowed"

144 embryos showed GFP-Daam1 localizing to the cell-cell junctions. However, we observed that

145 GFP-Daam1 also localized to cytoplasmic vesicles and cellular protrusions (Figure 2B, Video

146 S1). These observations are in line with previous reports on Daam1's localization and likely

147 hindered in imaging of fixed tissue due to unfavorable fixation conditions for observations of

148 cytoplasmic vesicles and cellular protrusions (Corkins et al., 2019; Jaiswal et al., 2013;

149 Kawabata Galbraith et al., 2018; Kida et al., 2007; Nishimura et al., 2012, 2016). Moreover, to

150 better understand the dynamics of Daam1 in the context of cell junctions, we imaged de novo

151 formation of cell-cell junctions in dissociated GFP-Daam1 expressing cells derived from the

152 nephric primordia (Figure 2A). In vivo time-lapse analyses of these cells showed that Daam1

153 localizes to filopodia-like protrusions and subsequently, to newly formed junctions (Figure 2B,

154 Video S2).

156 Finally, we assessed the localization of Daam1 in the mature epithelium of fully developed

157 nephrons (Figure S1). Interestingly and importantly, junctional localization of Daam1 was not

158 detected in the mature epithelium. Taken together, these data suggested the potential role for

159 Daam1 in regulating the intercellular adhesion of renal progenitors specifically at the onset of

160 tubulogenesis and CE. 


\section{Daam1 controls the organization and assembly of junctional F-actin within the nephric}

\section{3 primordium}

164 The observation that Daam1 co-localizes with junctional F-actin in developing Xenopus

165 nephron, led us to ask whether Daam1 regulates F-actin. To address this, we depleted Daam1

166 in nephron progenitors by utilizing Morpholino (MO) oligos in kidney-targeted microinjections

167 (DeLay et al., 2016; Moody and Kline, 1990). A proven Daam1 MO or an established Control

168 (standard) MO (Habas et al., 2001; Liu et al., 2008; Miller et al., 2011) was co-injected with a

169 membrane tagged GFP (mGFP) mRNA, that served as a linage tracer. MO-injected embryos were fixed at the onset of tubular morphogenesis (around NF stage 30) and subjected to whole-

171 mount fluorescent staining and high-resolution confocal imaging. To verify the success of our

172 injections and knockdowns, we also carried out Western blot analyses of total protein lysates

173 prepared from stage $30 \mathrm{MO}$-injected embryos (Figure 3B). Phalloidin staining revealed that

174 upon Daam1 depletion, the F-actin in renal progenitors becomes disorganized and significantly

175 reduced at cell-cell junctions (Figure $\mathbf{3 A}$ and $3 \mathrm{C}$, Videos S3 and S4). These results suggest

176 that Daam1 contributes to the organization of the F-actin cytoskeleton and actin filaments at

177 cell-cell junctions during nephron development.

179 We also observed alterations in spatial positioning and the overall organization of nephron

180 progenitors upon depletion of Daam1. Nephric primordia in Daam1-morphants consist of fewer

181 progenitor cells (Figure 3D) that are spaced farther part from one another (Figure 3E)

182 compared to control animals. In addition to changes in the number and position of nephron

183 progenitors, we also noted changes in cell morphology. Nephric progenitors with diminished

184 Daam1 activity display an increase in cell area (Figure 3F) and circularity (Figure 3G).

186 Since polymerization of F-actin filaments is a highly dynamic process, and to better understand 187 possible mechanisms underlying the observed morphological perturbations, we next assessed 
188 the dynamic behavior of F-actin in vivo using fluorescence recovery after photobleaching

189 (FRAP) assays. We labeled F-actin in developing nephrons by co-injecting mCherry tagged

190 Utrophin (mCherry-UtrCH) mRNA (Burkel et al., 2007) along with control or Daam1 morpholino.

191 To probe F-actin dynamics in the context of the intact animal, we utilized the "kidney-windowed"

192 approach in NF stage 30 embryos. Overall, FRAP experiments suggest slower turnover of

193 junctional F-actin in Daam1-depleted nephrons in comparison with controls (Figure 4). In

194 control nephrons, $100 \%$ of bleached cell junctions successfully recover fluorescence signal.

195 Whereas, in Daam1-depleted nephrons, only 59\% of bleached junctions recovered (Figure 4A).

196 Cell-cell junctions are comprised of dynamically mosaic E-cadherin clusters coupled to different

197 actin dynamics (Cavey et al., 2008; Indra et al., 2018). Therefore, the differences observed in

198 the dynamics of F-actin in Daam1 knockdown contexts potentially indicate the existence of two

199 actin pools differentially regulated by Daam1. However, it also possible that the observed

200 differences stem from the cell heterogeneity (e.g. in respect to Daam1 expression levels or cell-

201 type representation) within the nephric primordium. As we were unable to quantitatively assess

202 F-actin dynamics in junctions that fail to recover, we only used junctions with detectable

203 recovery signal to determine the mean half-time to recovery (Figure 4D) and the mean mobile

204 fraction (Figure 4E) values. For each junction values were taken from individually fitted curves.

205 These data indicate that the mean recovery half-time for junctional F-actin in Daam1 KD

206 nephrons is significantly slower (5.57 sec \pm 0.99 s.e.m.) in comparison with control nephrons

207 (3.70 sec \pm 0.35 s.e.m.) (Figure 4D). In contrast, the mean mobile fractions are relatively similar

208 (56.0\% \pm 4.2 s.e.m for control and $54.3 \% \pm 4.5$ s.e.m for Daam1 KD) (Figure 4E). From these

209 data, we conclude that Daam1 is driving the rate of F-actin turnover to promote polymerization

210 of junctional actin during nephron development. Moreover, these data also suggest that the

211 decrease in F-actin fluorescence levels observed upon Daam1 depletion is likely a

212 consequence of impaired actin assembly. 


\section{Daam1 promotes localization of E-cadherin at cell-cell contacts}

215 The interplay between E-cadherin and the actin cytoskeleton promotes intercellular adhesion

216 and assembly of cellular junctions. Therefore, we asked whether the changes observed in

217 junctional F-actin dynamics upon the knockdown of Daam1 alters the intercellular adhesion

218 between nephron progenitors. To examine if Daam1 regulates intercellular adhesion, we

219 analyzed the effect of Daam1 depletion on E-cadherin localization in developing nephron

220 (Figure 5). Daam1 morphants displayed reduced levels of E-cadherin at the interfaces between

221 neighboring cells during these early stages of pronephric morphogenesis (Figure 5A). This

222 difference was quantified and likewise made evident by measuring the fluorescence intensity

223 profiles of E-cadherin along the length of individual junctions (Figure 5B). Of note and in

224 contrast, there was no difference in the overall E-cadherin protein levels between Daam1

225 knockdown and control embryos as determined by Western blotting (Figure 5C). These findings

226 suggest that Daam1 is likely more important for localization of E-cadherin at cell-cell contacts as

227 opposed to regulating the overall expression levels of E-cadherin.

229 Interestingly, Daam1 depleted cells remain capable of forming nephrons. Studies carried out in

230 MDCK cells using the pan-formin inhibitor SMIFH2 suggest that formins are required for early,

231 but not later stages of cell-cell adhesion (Collins et al., 2017). Moreover, Daam1 depleted

232 mammary epithelial cells form a monolayer characterized by irregular tilting of lateral cell

233 membranes and distorted cell morphology (Nishimura et al., 2016). To further understand the

234 function of Daam1 in nephron assembly, we analyzed the epithelium of mature nephrons in NF

235 stages 39-40 embryos (Figure S2). Indeed, we did not observe any apparent changes in the

236 local concentration of junctional E-cadherin in mature nephrons of Daam1 knockdown and

237 control embryos (Figure S2A). However, Daam1 knockdown nephrons displayed defects in the

238 size of the tubular lumen. The diameter of tubular lumens was more variable in Daam1 deficient

239 nephrons compared to the controls. Similar to observations in mammary epithelial cells 
240 (Nishimura et al., 2016), tubular cells in Daam1 depleted nephrons are less uniform in shape

241 and characterized by an irregular tilting of lateral cell membranes (Figure S2A; Videos S5 and

242 S6). We further found that when visualized by transmission electron microscopy (TEM), Daam1

243 depleted cells appear less columnar, characterized by indistinct and wavy cell borders (Figure

244 S2B). Our results suggest that Daam1 regulates the adhesion between nephron progenitor cells

245 and subsequently, the morphology of the mature nephric epithelium.

247 E-cadherin localization is mediated by the Daam1 FH2 domain

248 Daam1 is known to act upstream of small Rho-GTPases, which regulate the actin cytoskeleton

249 (Habas et al., 2001; Liu et al., 2008); therefore, we next tested the importance of the actin

250 polymerization activity of Daam1 in intercellular adhesion. Formins are defined by a conserved

251 Formin Homology $2(\mathrm{FH} 2)$ domain. The Daam1 forms a dimer via its $\mathrm{FH} 2$ domain, responsible

252 for nucleation and elongation of actin filaments (Lu et al., 2007; Yamashita et al., 2007). The

253 mutation isoleucine-to-alanine (lle698Ala) in the Daam1 FH2 domain abolishes the actin

254 polymerization activity of Daam1 in vitro (Lu et al., 2007) and in vivo (Liu et al., 2008; Nishimura

255 et al., 2016). In kidney targeted-injections, we expressed either full-length GFP-Daam1 or GFP-

256 Daam1 FH2 mutant (Ile698Ala) mRNA and analyzed the effect on E-cadherin localization in the

257 nephric progenitors (Figure 6). Nephric progenitors expressing GFP-Daam1 FH2 mutant mRNA

258 showed reduced levels of E-cadherin at the interfaces between neighboring cells in comparison

259 to nephric progenitors expressing GFP-Daam1 (Figure 6A-B). However, the E-cadherin

260 phenotype appeared to be less prominent than in Daam1 knockdown. Functional studies

261 showed that while an isoleucine-to-alanine mutation within FH2 domain abolishes Daam1's

262 ability to polymerize actin, it does not prevent its activation of Rho (Liu et al., 2008). This could

263 be one possible explanation as to why E-cadherin localization is more affected in Daam1

264 knockdown nephric progenitors. Furthermore, we assessed Daam1 protein levels in injected

265 embryos to determine if the observed differences in E-cadherin localization were potentially due 
266 to underlying disparities in translation efficiency or protein stability of GFP-Daam1 and GFP-

267 Daam1 FH2 mutant (Figure 6C). We found that Daam1 protein was present at equivalent levels 268 in the two samples, making these possibilities unlikely. Ultimately, the GFP-Daam1 FH2 mutant 269 expressing progenitors mature into nephrons characteristic of Daam1 knockdown (Figure S3).

270 These results establish that the Daam1 FH2 domain is necessary for the localization of E-

271 cadherin to cell-cell contacts in nephron progenitors.

272

\section{Daam1 mediates cohesion of MDCK cells}

274 Nephron morphogenesis is achieved through the process called convergent extension (CE)

275 (Lienkamp et al., 2012). The CE is a type of collective cell movement characterized by a series

276 of coordinated and directed cell rearrangements (Huebner and Wallingford, 2018; Tada and

277 Heisenberg, 2012). In recent years E-cadherin has emerged as a key mediator for coordinating

278 cohesion and directional persistence of collective cell movements in both epithelial and

279 mesenchymal clusters (Cai et al., 2014; Campbell and Casanova, 2015; Cohen et al., 2016).

280 Our results demonstrate that Daam1 is necessary for the organization of nephrogenic

281 primordium (Figure 3) and a proper localization of E-cadherin (Figures 5 and 6); therefore, we

282 wanted to determine if Daam1 is necessary for the coordination of direction between renal cells.

283 Because the opaqueness of Xenopus nephron progenitors prevents in vivo tracking of their

284 movements in 3D, we utilized Madin-Darby Canine Kidney (MDCK) cells. We generated MDCK

285 cells constitutively expressing an shRNA against Daam1 and analyzed whether the E-cadherin

286 localization is affected in these cells (Figures 7A and S4). The efficiency of shDaam1

287 knockdown was confirmed by Western blot (Figure 7B). Similar to what we saw in Xenopus

288 nephrons, we observed impaired localization of E-cadherin in nascent (Figure 7A), but not

289 mature adhesions (Figure S4) upon knockdown of Daam1. We next examined the migratory

290 behavior of Daam1 knockdown cells in a time-lapse imaging of the wound-healing assay

291 (Figure 7C-F; Video S7). We found Daam1 knockdown cells exhibit a delay in a wound closure 
292 compared to control cells (Figure 7C; Video S7). Additionally, we also observed random

293 detachment of Daam1-deficient cells from the migrating epithelial sheets (Video S7). To better

294 understand the behavior of these cells, we tracked their movement within the migrating sheets

295 over time. (Figure S7). From these tracks, we obtained the relative distances over which cells

296 traveled and used those distances to assess cell velocities (Figure 7C). These analyses show

297 that the speed at which Daam1 knockdown cells move is higher than that of the control cells,

298 demonstrating that delayed wound closure in Daam1 knockdown cells is not caused by their

299 slow movement. However, mapping the trajectory paths for Control (Figure 7E) and shDaam1

300 (Figure 7D) cells revealed that the movement of Daam1-deficient cells is less directed

301 compared to control. These data demonstrate that Daam1 is necessary for communication of

302 direction between the cells. Taken together, these results indicate that Daam1 contributes to

303 cohesion by regulating connectiveness of cells through E-cadherin.

\section{DISCUSSION}

306 Using Xenopus embryonic kidney and MDCK cells as model systems, we show that Daam1

307 mediates E-cadherin dependent intercellular adhesion and organization of nephrogenic

308 primordia by regulating polymerization of junctional actin filaments. Ultimately, this affects the

309 morphology of mature nephric epithelium. These findings have a number of important

310 implications for the regulation of intercellular adhesion and epithelial tubulogenesis.

312 First, we show that both Daam1 and E-cadherin localize to cell-cell contacts during nephron

313 development and that Daam1 is required for promoting E-cadherin localization at sites of cell-

314 cell contact. In contrast to what we observed during these early stages of nephron development

315 following the depletion of Daam1, in mature nephrons, we were unable to detect junctional

316 Daam1, and we likewise did not observe the effect on localization of E-cadherin. These data 
317 suggest that Daam1 is necessary for efficient localization of E-cadherin at cell junctions in

318 nephron progenitor cells during early stages of nephron morphogenesis.

320 This conclusion builds upon certain assumptions. For example, it is possible that the

321 overexpression of GFP-Daam1 has an impact on our localization analyses, or that cells

322 depleted of Daam1 in mature nephrons are starting to recover due to a decrease in available

323 morpholino pools with time. However, multiple lines of evidence suggest that both of these

324 scenarios are highly unlikely. In the first case, prior studies indicate that the overexpression of

325 full length Daam1 has little to no affect the actin cytoskeleton or other cellular processes (Liu et

326 al., 2008). Additionally, several studies demonstrate that the distribution of the GFP-Daam1

327 recapitulates its endogenous localization as discerned via immunostaining (Jaiswal et al., 2013;

328 Li et al., 2011; Nishimura et al., 2012, 2016). In the second case, consistent with our

329 observations in Xenopus nephrons, sub-confluent cultures of MDCK cells stably expressing

330 shDaam1 show transient repression of E-cadherin localization to cell-cell contacts that appears

331 to be lost as the cells become confluent.

333 Indeed, our data support recent findings suggesting that actin nucleating proteins and Rho

334 GTPases are required for early stages of E-cadherin mediated cell-cell adhesion in MDCK

335 epithelial cells and not the maintenance of mature junctions (Collins et al., 2017). They also

336 imply that morphological defects that we see in mature nephrons are consequences of earlier

337 events. It is interesting to note that in similar fashion the silencing of E-cadherin expression in

338 MDCK cells disrupts formation of cell-cell junctions whereas its signaling seems to be largely

339 dispensable in already established epithelium as long as the cells are not mechanically stressed

340 (Capaldo and Macara, 2007). 
342 Second, we show that Daam1 localizes to actin protrusions and newly formed cell-cell contacts

343 in developing nephron, suggesting that Daam1 via E-cadherin coordinates the assembly of cell-

344 cell junctions during CE. Actin polymerization at the cell's membrane mediates polarized

345 movement and intercalation of cells during CE through engagement of cadherins (Huebner and

346 Wallingford, 2018; Huebner et al., 2020). Here, we show that Daam1 activity functions to ensure

347 proper organization and size of nephrogenic primordium at the time of CE as well as polarized

348 movement of renal epithelial sheets. Our data support previous studies implicating Daam1 in CE

349 and polarized cell movements (Ang et al., 2010; Kida et al., 2007; Liu et al., 2008). Furthermore,

350 we show that Daam1 mediates polarized movement and cohesion of MDCK cells without

351 slowing down the motility of individual cells. Taken together these data suggest that Daam1

352 promotes collective cell movements by controlling actin's polymerization at cell-cell contacts and

353 strengthening of E-cadherin-based adhesion.

355 Finally, Wnt9b and Wnt11 regulate tubular nephron morphogenesis, and disruptions in the cell

356 behaviors traditionally regulated by the PCP pathway define their loss-of-function phenotypes

357 (Karner et al., 2009; O'Brien et al., 2018). We show that inhibiting signaling activity of Daam1 in

358 the prospective nephron results in a set of phenotypic characteristic comparable to those

359 reported for Wnt11 and Wnt9b. Moreover, earlier studies have demonstrated that Daam1 can

360 rescue Wnt11- induced CE gastrulation defects (Liu et al., 2008). These data collectively point

361 to Daam1 as a potential downstream effector of Wnt11 and Wnt9b signaling in the control of

362 nephric tubulogenesis. Interestingly, wnt9b and wnt9a are also induced in the injured nephrons

363 and a mutation in $f z d 9 b$ is associated with reduced regenerative capacity of nephric tubules

364 (Kamei et al., 2019). These findings also suggest a potential role for Daam1 in repair and

365 regeneration of nephric tubules. This hypothesis is further supported by increasing research

366 evidence that the actin-based protrusions are important for the repair of cell-cell junctions (Li et 
367 al., 2020) and studies showing that Daam1 promotes assembly of actin-based protrusions

368 (Jaiswal et al., 2013; Nishimura et al., 2016).

370 Cadherin localization is a complex process, and current studies propose at least three different

371 ways of achieving efficient localization of E-cadherin: (1) clustering enforced by cortical F-actin,

372 (2) clustering regulated by exposure of neighboring cells through their actin-based protrusions

373 and (3) clustering promoted by condensation of F-actin networks via myosin (Yap et al., 2015).

374 However, further research into timely regulation of E-cadherin is required to characterize the

375 precise molecular details and specify relationships between different modes of E-cadherin

376 clustering. Ultimately, investigations into how regulation of junctional E-cadherin by Daam1 fits

377 in with different modes of E-clustering will also be important to examine in the future.

\section{ACKNOWLEGMENTS}

380 We thank Miller lab members as well as Dr. Pierre McCrea and Dr. Jae-II Park and their lab

381 members for lively discussions and suggestions on the manuscript. We also thank Dr. Richard

382 Behringer, Dr. Yoshihiro Komatsu, Dr. Oleh Pochynyuk and Dr. Anna Marie Sokac, for their

383 input and suggestions on the project. We thank Kenneth Dunner Jr. at the UT MD Anderson

384 High Resolution Electron Microscopy Facility and CCSG grant NIH P30CA016672 for support

385 with the transmission electron microscopy data. We are also thankful to Raymond Habas, Bruce

386 Goode, Norihiro Sudou and Masanori Taira for providing antibodies and constructs. We thank

387 the instructors and teaching assistants of the 2015 National Xenopus Resource Advanced

388 Imaging Course. We are grateful to J.C. Whitney and T.H. Gomez who took care of the animals,

389 even during Hurricane Harvey. We are grateful to the UTHealth Office of the Executive Vice

390 President and Chief Academic Officer and the Department of Pediatrics Microscopy Core for

391 funding the Zeiss LSM800 confocal microscope. We also thank BSRB Microscopy Facility at

392 Department of Genetics, UT-MD Anderson Cancer Center. This work was funded by National 
Institute of Diabetes and Digestive and Kidney Diseases grants (K01DK092320, R03DK118771 and R01DK115655 to R.K.M.), startup funding from the Department of Pediatrics, Pediatric Research Center at the McGovern Medical School (to R.K.M.), The Antje Wuelfrath Gee and Harry Gee, Jr. Family Legacy Scholarship (to V.K.S.), and The Gigli Family Endowed

397 Scholarships (to V.K.S.)

\section{AUTHOR CONTRIBUTIONS}

400 V.K.S. conceived of the project, preformed experiments, analyzed data and wrote the original 401 draft of the manuscript. M.E.C. cloned pCS2-mCherry-Daam1 and pCS2-mCherry-Daam1 402 (Ile698Ala) constructs and contributed to data validation. V.K.S., M.E.C., A.B.G. and R.K.M. 403 preformed experiments to generate MDCK shDaam1 cells. V.K.S. and A.P. imaged the wound 404 healing assays, FRAP experiments and analyzed FRAP data. V.K.S. and M.K. conducted TEM 405 imaging analyses. A.B.G. and R.K.M. oversaw the experiments and supervised the project. All 406 authors were involved in critical evaluation and editing of the manuscript.

\section{DECLARATION OF INTERESTS}

The authors declare no competing interests.

\section{FIGURE TITLES AND LEGENDS}

Figure 1. Daam1 co-localizes with junctional F-actin and E-cadherin during early nephron development primordium labeled by Lhx1(magenta) and GFP to visualize Daam1 (green) in conjunction with, (A) Phalloidin staining to visualize F-actin (red) or

(B) E-cadherin (cyan);

a'-a"' and b'-b"' represent close-up images of white boxes. 
427 Figure 2. Daam1 localizes to newly formed cell-cell contacts

428 (A) Schematic illustration showing steps involved in preparation of "windowed kidney" embryos

429 and primary cultures expressing GFP-Daam1. Please note that for clarity of illustration the 8-cell

430 GFP-Daam1 injected Xenopus blastomere is fate-mapped strictly to the nephric primordium and

431 that blastomere also contributes to epidermis, ventral and dorsal somites, hidgut, proctodeum

432 and trunk neural crest cells (DeLay et al., 2016; Moody and Kline, 1990).

433 (B) Time-lapse imaging montage of the nephric primordium expressing GFP-Daam1 in

434 "windowed kidney" embryos. Elapsed time is indicated at the top in seconds; see Video S1.

435 (C) Time-lapse imaging montage shows cells isolated from a developing nephron expressing 436 GFP-Daam1 mRNA adhering with each other. Elapsed time is indicated at the top in seconds.

437 The border arising between two cells is delineated by the red dotted line; see Video S2.

438

\section{Figure 3. Effects of Daam1 depletion on the nephrogenic primordium}

(A) Maximum projection confocal images of F-actin expression (red) in nephric primordium (magenta) in Control and Daam1 knockdown embryos. a-a" and b'-b" represent close-up images of the corresponding regions in white boxes; see Videos S3 and S4.

(B) Western blot showing Daam1 and GAPDH (control) protein levels for uninjected wild type (WT) and Control (Standard morpholino) and Daam1 KD (Daam1 morpholino) injected embryos.

(C) The graph showing the relative fluorescence intensity levels of junctional F-actin in the nephric primordia of Control and Daam1 KD embryos. Ncontrol=40 junctions on 2 embryos and NDaam $1 \mathrm{KD}=40$ junctions on 2 embryos. ${ }^{* * * *} \mathrm{P}<0.0001$ analyzed by unpaired t-test.

(D-G) Morphometric analyses of Control and Daam1-depleted nephric primordia. The thick bars represent the mean, ${ }^{* * * *} \mathrm{P}<0.0001$ analyzed by unpaired $t$-test, $(E-G)$ Ncontrol=40 junctions on 2 embryos and NDaam 1 KD=40 junctions on 2 embryos. Graphs showing comparison between

\section{Control and Daam1-depleted nephric primordia of,}

(D) the average number of Lhx1-positive nephron progenitors where $\mathrm{N}$ control=5 embryos and NDaam1KD=5 embryos,

(E) the relative distance between nearest neighbors of Lhx1-positive nuclei, 
$(\mathrm{F})$ the relative cell area and

(G) the relative circularity, where 1 represents the perfect circle.

\section{Figure 4. Daam1 regulates assembly of junctional F-actin in developing nephron}

F-actin dynamics at cell-cell junctions of Control and Daam1-depleted developing nephrons expressing mCherry-Utrophin were assessed using FRAP.

(A) Percentage of junctions showing recovery of fluorescence after bleaching in Control (black, Ntotal=27 junctions, 1-5 junctions/embryo) and Daam1 KD (purple and orange, Ntotal=27 junctions, 1-5 junctions/embryo) nephrons.

(B) Typical time-lapse images of Control and Daam1-depleted cell junctions before and after photobleaching. In each image, the bleached region is highlighted with a circle (black - Control junction showing recovery, purple - Daam1 KD junction showing recovery and orange - Daam1 $\mathrm{KD}$ junction showing no-recovery of fluorescence after photobleaching).

(C) Graph shows average recovery curves obtained from individual best-fit plots for Control (black), Daam1 KD junctions with (purple) and without (orange) recovery of fluorescence after photobleaching.

(D-E) Bar graphs comparing Control and Daam1 KD profiles calculated from individual best-fit curves for Control (black) and Daam1 KD junctions with recovery of fluorescence after photobleaching (purple). Data represent the mean \pm S.E. from three independent experiments. P-values were analyzed by unpaired t-test.

(D) Bar graph of the relative half-times for F-actin.

(E) Bar graph of the relative mobile fraction for $\mathrm{F}$-actin.

\section{Figure 5. Daam1 promotes localization of junctional E-cadherin}

(A) Maximum projection confocal images of E-cadherin expression (cyan) in nephric primordium (magenta) in Control and Daam1 KD embryos a-a' and b-b' represent the close-up images of corresponding regions in white boxes. 
(B) Violin plots depicting the relative fluorescence intensity of junctional E-cadherin in the nephric primordia of Control (orange) and Daam1 KD (blue). Ncontrol=88 junctions on 4 embryos and NDaam 1 KD=84 junctions on 4 embryos. Center-lines represents median; Limits show 1 st and 3rd quartile. ${ }^{\star \star * *} \mathrm{P}<0.0001$ analyzed by unpaired t-test.

(C) Western blot showing Daam1, E-cadherin and GAPDH (control) protein levels in uninjected wild type (WT) and Control (Standard morpholino) and Daam1 KD (Daam1 morpholino) injected embryos. Daam1

(A) Maximum projection confocal images showing E-cadherin staining (cyan) in nephric primordium (magenta) expressing GFP-Daam1 or GFP-Daam1 FH2 mutant mRNA. a-a' and bb' represent close-up images of corresponding regions in white boxes.

(B) Violin plots depicting the relative fluorescence intensity of junctional E-cadherin in the nephric primordia expressing GFP-Daam1 (orange) and GFP-Daam1 FH2 mutant (blue) mRNA. NDaam1 $=60$ junctions on 3 embryos and NDaam1FH2mutant=55 junctions on 3 embryos. Center-lines represents median; Limits show 1 st and 3rd quartile. ${ }^{\star \star \star} \mathrm{P}<0.0002$ analyzed by unpaired t-test.

(C) Western blot showing the exogenous and endogenous protein levels of Daam1 in uninjected wild type (WT) embryos, embryos injected with $1 \mathrm{ng}$ of GFP-Daam1 mRNA and 1ng GFPDaam1 FH2 mutant mRNA. The non-specific band confirms equal loading.

\section{Figure 7. Daam1- depleted MDCK cells display compromised localization of E-cadherin at} cell-cell contacts and impaired cohesion during collective movement

(A) E-cadherin (green), F-actin (red) and DAPI (blue) in subconfluent the MDCK Control and

512 shDaam1 knockdown cells. E-cadherin localization in the nascent cell-cell contacts (marked by

513 white brackets and shown enlarged in corresponding white boxes) is impaired in shDaam1-

514 deficient cells.

515 (B) Western blot analysis of Daam1 and GAPDH protein levels in the MDCK Control and 516 shDaam1 knockdown cells.

517 (C-F) Summary of the wound-healing experiments for the MDCK Control and Daam1 KD cells, 518 see Video S7. 
519 (C) Daam1 depletion impairs wound closure. The graph represents the percent of the wound 520 surface area over time for Control (purple) and Daam1 KD (orange) cells. Error bars indicate 521 S.E. of the mean on 4 assays.

522 (D-F) Manually tracking migration paths of single-cells during the wound closure demonstrates 523 that Daam1 organizes collective movement of the MDCK epithelial monolayers by modulating 524 the speed and directionality of individual cells. Depletion of Daam1 results in increased velocity 525 and random migration. Ncontrol=52 cells from 4 assays and NDaam $1 \mathrm{KD}=42$ cells tracked from 4 526 assays. Cells were tracked in 15 minutes increments for 12 hours.

527 (D) Violin plots represent migration velocity calculated from tracking traveled distances of single 528 cells for Control and Daam1 KD cells. Center-lines represents median; Limits show 1 st and 3rd 529 quartile. ${ }^{* *} \mathrm{P}<0.0001$ analyzed by unpaired t-test.

530 (E) Wind-rose plot showing migration tracks of individual Control cells.

531 (F) Wind-rose plot showing migration tracks of Daam1 KD cells.

MATERIALS AND METHODS

\section{Xenopus laevis}

536 Xenopus laevis adult male and female frogs were obtained from Nasco (LM00531MX and

537 LM00713M) (Fort Atkins, WI, USA) and maintained according to standard procedures. Xenopus

538 embryos were obtained by in vitro fertilization (Sive et al., 2000) and staged as previously

539 described by Nieuwkoop and Faber (NF) (Nieuwkoop and Faber, 1994). All work was carried

540 out in accordance with the University of Texas Health Science Center at Houston, Institutional

541 Animal Care and Use Committee (IACUC) protocol \#AWC-19-0081.

\section{$543 \quad$ MDCK cell lines}

544 Madin-Darby Canine Kidney (MDCK) II cell lines were purchased from the American Type 545 Culture Collection (ATCC). MDCK cells were cultured at $37^{\circ} \mathrm{C}$ with $5 \%$ CO2 in Dulbecco's 
546 Modified Eagle's Medium (DMEM) (Sigma, D6429) supplemented with 10\% fetal bovine serum

547 (FBS) (Sigma, F0926) and 1\% Antibiotic-antimycotic solution (Sigma, A5955).

549 Embryo microinjections

550 Xenopus embryos were microinjected at one-cell or into V2 blastomere at eight-cell stage,

551 targeting embryonic kidney (DeLay et al., 2016; Moody and Kline, 1990; Nieuwkoop and Faber,

552 1994). Embryos were injected with synthetic mRNAs alone or in combination with antisense

553 morpholino oligonucleotides (MOs). For mRNA injections, capped mRNA transcripts were

554 synthesized from DNA-plasmids using SP6 mMessage mMachine transcription kit

555 (ThermoFisher, AM1340M) and purified. pCS2-GFP-Daam1 and pCS2-GFP-Daam1 (Ile698Ala)

556 plasmids were a gift from Dr. Raymond Habas's and Dr. Bruce Goode's labs, respectively (Lu et

557 al., 2007). A mutation A2822G discovered in these plasmids was corrected by site directed

558 mutagenesis as previously reported (Corkins et al., 2019) prior to mRNA synthesis. pCS2-

559 membrane-tagged-RFP (mRFP)(Davidson et al., 2006), pCS2-membrane-tagged-EGFP

560 (mEGFP)(Shindo and Wallingford, 2014) and pCS2-mCherry-Utrophin (mCherry-UtrCH)(Burkel

561 et al., 2007) constructs were gifts from Dr. Raymond Keller's lab, Dr. John Wallingford's lab and

562 Dr. William Bement's lab, respectively. Formerly developed translation-blocking Daam1

563 (5'GCCGCAGGTCTGTCAGTTGCTTCTA 3') (Corkins et al., 2018; Habas et al., 2001; Miller et

564 al., 2011) and standard control (5'CCTCTTACCTCAGTTACAATTTATA 3') MOs were

565 purchased from GeneTools, LLC (Philomath, OR, USA). MOs were injected at 20ng per embryo

566 while the amount of injected mRNA per embryo were as follows: GFP-Daam1 [1ng], mCherry-

567 Daam1 [1ng], GFP-Daam1(I698A) [1ng], mCherry-Daam1(I698A) [1ng], mRFP [0.5ng], mGFP

568 [0.5ng] and mCherry-UtrCH [1ng].

570 Generation of stable MDCK shDaam1 cell lines 
571 shDaam1 knockdown cell lines were generated by a retrovirus-based transduction method as

572 described (Corkins et al., 2019). Briefly, the pLKO.1 lentiviral shDaam1 constructs

573 (TTTCAGGAGATAGTATTGTGC, AAACAGGTCTTTAGCTTCTGC) were purchased from GE-

574 Dharmacon (Clone ID: TRCN0000122999, Clone ID: TRCN0000123000). HEK293T cells were

575 co-transfected with shDaam1 and virus packaging plasmids (psPAX2 and pMD2.G) using

576 Polyethylenimine (PEI). The viral titers were collected starting 24 hours post transfection over

577 the course of two days and purified using $0.22 \mu \mathrm{m}$ Polyethersulfone (PES) syringe filters.

578 Infections were carried out in the presence of polybrene. MDCK II cells remained in infection

579 media for 24 hours, followed by puromycin selection with final concentration of $0.70 \mu \mathrm{g} / \mathrm{ml}$.

580 Lastly, MDCK II shDaam1 knockdown stable cell lines were validated by Western blotting.

\section{$582 \quad$ Western blotting}

583 Western blotting was carried out using published protocols (Kim et al., 2002; Williams et al., 584 2017). In short, Xenopus embryos were cultured to desired stage and collected. The whole-

585 embryo lysates were prepared by resuspending 10-20 embryos in a prechilled TX100-lysis

586 buffer (10 mM HEPES, 150 mM NaCl, 2 mM EDTA, 2 mM EGTA, 0.5\% Triton X-100, pH 7.4)

587 and centrifuged at $18,407 \mathrm{RCF}$ at $4^{\circ} \mathrm{C}$ for 5 minutes. The resulting protein lysates were

588 resuspended in an equal volume 2X Laemmli (BioRad,161-0737) + dithiothreitol (Fisher

589 BioReagents, $\mathrm{BP} 17225)$ solution, and incubated at $95^{\circ} \mathrm{C}$ for 2 minutes. For making protein

590 lysates using MDCK cells, cells were washed with PBS and pelleted by centrifugation at 18,407

$591 \mathrm{RCF}$ at $4^{\circ} \mathrm{C}$ for 5 minutes. Cell pellets were resuspended in in a prechilled Triton-lysis buffer (50

$592 \mathrm{mM}$ Tris $\mathrm{pH}$ 7.4, 1\% Triton X-100, $150 \mathrm{mM} \mathrm{NaCl}, 1$ mM EDTA, 1 mM EGTA, 1 mM PMSF, 1 mM

$593 \mathrm{Na}_{3} \mathrm{VO}_{4}, 10 \mathrm{mM}$ sodium fluoride, $10 \mathrm{mM} \beta$-glycerophosphate, $1 \mathrm{mg} / \mathrm{ml}$ aprotinin, and $1 \mathrm{mg} / \mathrm{ml}$

594 leupeptin). The cell lysates were incubated on ice for 20 minutes, followed by sonication and

595 centrifugation at $18,407 \mathrm{RCF}$ for 10 minutes at $4^{\circ} \mathrm{C}$. Bradford assay was used to determine the 
596 total amount of protein in the lysates. The protein samples were run on an $8 \%$ SDS-PAGE gel

597 and transferred to nitrocellulose or polyvinylidene difluoride (PVDF) membranes. The blots were

598 blocked for at least 1.5 hours at the room temperature using the KPL Detector Block Kit (Sera

599 Care, 5920-0004, 71-83-00) and probed with primary antibodies overnight at $4^{\circ} \mathrm{C}$. The next day,

600 after series of washes with Tris-buffered saline containing $0.1 \%$ Tween 20 (TBST), blots were

601 incubated with secondary antibodies for at least 1 hour at the room temperature. Protein

602 expression levels were detected with enhanced chemiluminesence (SuperSignal West Pico

603 PLUS Chemiluminescent Substrate, Thermo Fisher, 34580) using LiCor and BioRad ChemiDoc

604 XRS imagers. The following antibodies were used: rabbit anti-Daam1 (1:1000, Proteintech,

605 14876-1-AP), rabbit anti-Daam1 (1:1000, gift from Dr. Raymond Habas), rabbit anti-GAPDH

606 (1:1000, Santa Cruz, sc-25778), mouse anti-E-cadherin (1:1000, BD Transduction Laboratories, 607 610182), rabbit anti-GFP (1:250, ICL Lab, RGFP-45A), anti-rabbit IgG (H + L)-HRP (1:5000,

608 BioRad,1706516) and anti-mouse IgG (H + L)-HRP (1:5000, BioRad, 1706516).

\section{Immunostaining and staining}

611 Xenopus embryos were fixed in MEMFA (3.7\% formaldehyde, 4 mM MOPS, 2 mM EGTA, and 1 $612 \mathrm{mM} \mathrm{MgSO}_{4}, \mathrm{pH} 7.4$ ) for 1 hour at room temperature or overnight at $4^{\circ} \mathrm{C}$. Embryos to be stained 613 with Phalloidin, were fixed using methanol-free formaldehyde (Thermo Scientific, 28908).

614 Immunostaining was carried out according to previously published methods (Hemmati-Brivanlou 615 and Melton, 1994; Krneta-Stankic et al., 2010). In short, fixed-embryos were washed 3 times for 61615 minutes at room temperature with phosphate buffered saline (PBS) containing $0.1 \%$ Triton X617100 and $0.2 \%$ bovine serum albumin (BSA) and blocked using $10 \%$ goat serum diluted in PBST 618 for 1 hour at room temperature. Embryos were incubated with the primary antibodies overnight

619 at $4^{\circ} \mathrm{C}$. The next day, embryos were washed 5 times for 1 hour with PBS-T at room temperature 620 and then incubated with the secondary antibodies overnight at $4^{\circ} \mathrm{C}$. The following day, embryos 
621 were washed 3 times for $1 \mathrm{hr}$ at room temperature and dehydrated in methanol prior to clearing.

622 To preserve Phalloidin-labeling, isopropanol was used to dehydrate Phalloidin-stained embryos

623 (Nworu et al., 2014). Embryos were cleared using BABB (1-part benzyl alcohol: 2-parts benzyl

624 benzoate) clearing solution and imaged. MDCK cells were fixed in 4\% paraformaldehyde (PFA)

625 for 10 minutes at room temperature. Cultures were washed 3 times with PBS and incubated

626 with $50 \mathrm{mM}$ ammonium chloride for 10 minutes at room temperature to neutralize the PFA. Next,

627 samples were washed 3 times with PBS and blocked using 10\% goat serum/ PBST for 1 hour

628 and incubated with primary antibodies overnight at $4^{\circ} \mathrm{C}$. The following day, MDCK cells were

629 washed 3 times with PBS and incubated with secondary antibodies for 1 hour at room

630 temperature. Lastly, stained samples were washed again 3 times with PBS prior to mounting in

631 Fluoromount-G medium (Southern Biotech, 0100-01) for imaging. The following primary

632 antibodies were used: chicken anti-GFP (1:250, Abcam, ab13970), rabbit anti-RFP (1:250, MBL

633 International, PM005), rabbit anti-GFP (1:250, ICL Lab, RGFP-45A), rabbit anti-Lhx1 (1:250, gift

634 from Dr. Masanori Taira) and mouse anti-E-cadherin (1:100, BD Transduction Laboratories,

635 610182). For detection of primary antibodies the following secondary antibodies were used:

636 anti-rabbit IgG Alexa 488 (1:500, Invitrogen, A-11008), anti-rabbit IgG Alexa 555 (1:500,

637 Invitrogen, A-21428), anti-rabbit IgG Alexa 647 (1:500, Invitrogen, A-21244), anti-mouse IgG

638 Alexa 488 (1:500, Invitrogen, A-11001), anti-mouse IgG Alexa 555 (1:500, Invitrogen, A-21422),

639 anti-mouse IgG Alexa 647 (1:500, Invitrogen, A-21235), anti-mouse IgG Alexa 488 (1:500,

640 Jackson ImmunoResearch, 715-545-150) and anti-chicken IgY Alexa 488 (1:500, Invitrogen, A-

641 11039). Fluorescent probes used for staining were as follows: FITC-conjugated lectin from

642 Erythrina cristagalli (1:500, Vector labs, FL-1141), Phalloidin-Alexa 568 (embryos- 1:40 and

643 cells-1:200, Invitrogen, A12380), and diamidino-2-phenylindole (DAPI) (1:500, Thermo

644 Scientific, 62247).

645

646 Transmission electron microscopy (TEM) 
647 Xenopus embryos were fixed in 2\%formaldehyde+0.5\% glutaraldehyde (Ted Pella Inc., 18505

648 and 18426). Fixed embryos were washed with $0.1 \mathrm{M}$ sodium cacodylate buffer and treated with

$649 \quad 0.1 \%$ Millipore-filtered cacodylate buffered tannic acid. Embryos were post-fixed using $1 \%$

650 buffered osmium, followed by staining using 1\% Millipore-filtered uranyl acetate. Stained

651 embryos were dehydrated by washing in increasing concentrations of ethanol, permeated, and

652 embedded in LX-112 medium. After embedding, embryos were placed in a $60^{\circ} \mathrm{C}$ oven for

653 approximately 3 days to polymerize. Polymerized samples were sectioned using Leica Ultracut

654 microtome (Leica, Deerfield, IL). Collected ultrathin sections were stained with uranyl acetate

655 and lead citrate in a Leica EM Stainer and subjected to imaging.

657 Isolation and live imaging of Xenopus kidney cells

658 Embryos were microinjected with 1ng GFP-Daam1 mRNA into the V2 blastomere, targeting 659 kidney. Around embryonic NF stage 30, GFP-positive nephrons were surgically dissected under 660 a fluorescent stereomicroscope using a pair of sharpened forceps (Fisher, NC9404145).

661 Microsurgical dissections were performed in plastic petri dishes coated with $2 \%$ agar containing 662 Danilchik's for Amy (DFA) solution (53 mM NaCl, 5 mM Na2 $\mathrm{CO}_{3}$, $4.5 \mathrm{mM}$ Potassium Gluconate, $66332 \mathrm{mM}$ Sodium Gluconate, $1 \mathrm{mM} \mathrm{CaCl}_{2}, 1 \mathrm{mM} \mathrm{MgSO}_{4}$, buffered to $\mathrm{pH} 8.3$ with $1 \mathrm{M}$ bicine )

664 supplemented with $1 \mathrm{~g} / \mathrm{l}$ of and Antibiotic antimycotic solution (1:100, Sigma, A5955). To

665 dissociate into single cells, the isolated nephrons were transferred to fibronectin coated $(1 \mu \mathrm{g} / \mathrm{ml}$,

666 Roche, 10-838-039-001) glass-bottom imaging chambers (Thermo, A7816) prefilled with

667 Calcium Magnesium Free Media (CMFM) (88 mM NaCl, 1 mM KCl, 2.4 mM NaHCO , 7.5 mM

668 Tris $\mathrm{pH} 7.6)$. After $\sim 30$ minutes at the room temperature, as much as possible of the CMFM

669 media was aspirated from top of the chamber without disturbing the cells. Fresh DFA media was

670 added and again removed by careful aspiration. In order to ensure complete removal of the 
671 CMFM media, this process was repeated at least 5 times. The cells were left undisturbed at the

672 room temperature for $15-30$ minutes prior to imaging.

\section{Wound-healing assay}

675 MDCK cells were seeded in 6-well culture plates at a density of 100,000 cells/well and allowed

676 to reach confluency. Prior to inducing a "wound" or scratch or in a confluent cell monolayer, the

677 cells were treated with Mitomycin C $(10 \mu \mathrm{g} / \mathrm{ml})$ for 3 hours at $37^{\circ} \mathrm{C}$ to prevent future proliferation.

678 A linear scratch was made by gliding the $200 \mu$ sterile tip across the bottom of each well. After

679 making a scratch, cells were washed with 1X PBS and refed with $5 \mathrm{ml}$ of 10\%FBS/DEMEM

680 supplemented with $1 \%$ Antibiotic-antimycotic solution. Samples were placed in $37^{\circ} \mathrm{C}$ heated

681 imaging chamber with 5\% CO2 and subjected to time-lapse imaging.

\section{Fluorescence Recovery after Photobleaching (FRAP)}

684 To visualize actin dynamics, embryos were injected into V2 blastomere at 8-cell stage with $1 \mathrm{ng}$ 685 mCherry-UtrCH mRNA along with 20 ng of Daam1 or Standard (control) MO. Injected embryos were cultured to around NF stage 30. Since the opaqueness of Xenopus epithelium prevents

687 direct in vivo imaging of developing nephron at this early embryonic stage, "windowed" embryos 688 were generated. To make windowed embryos, embryos were anesthetized in $0.04 \%(0.15 \%)$

689 Ethyl 3-aminobenzoate methanesulfonate (Sigma, E10521) diluted in DFA. Next, the epithelium 690 covering developing nephron was surgically removed under fluorescence-dissecting

691 microscope, exposing developing nephron for in vivo imaging. Windowed embryos were

692 mounted under glass-cover slips and subjected to FRAP. FRAP assays were performed on an

693 inverted 3i spinning disk microscope integrated to a NIKON TiE with perfect focus and equipped

694 with Vector ${ }^{\text {TM }}$ FRAP scanning module and a Hamamatsu Flash 4.0 camera. Images were

695 acquired with a NIKON Plan-Apo 60X water 1.2 NA objective. A small fragment at the midpoint 
696 of a cell-cell junction was bleached with the $561 \mathrm{~nm}$ laser line at $100 \%$ power. A series of twenty

697 pre-bleach images were captured and post-bleach recovery was recorded continuously until

698 fluorescent signal reached a steady state. Movies were analyzed using Slidebook 6.0 and curve

699 fitting was done with SigmaPlot and GraphPad Prism 8.0 software. For curve fitting, a single

700 exponential function $\left(f(t)=\alpha(1-e-k T)\right.$, where $T_{1 / 2}$ (half-time of recovery) is $\ln 0.5 /(-k)$, and $\alpha$ is the

701 mobile fraction was used. Raw recovery curves were corrected for background and photofading.

702 Lowest fluorescence signal and the time-point after bleaching were scaled to 0 and curves were

703 normalized to 1 based on the reference signal before bleaching. Per embryo, between 1 and 5

704 single junctions were photobleached. To avoid bleaching-induced variation in fluorescence,

705 junctions picked for photobleaching were spaced far apart. All FRAP experiments have been

706 carried out using multiple embryos and repeated at least three times.

\section{Image acquisition and processing}

709 Olympus SZX16 fluorescent stereomicroscope equipped with Olympus DP71 camera was used

710 for carrying out Xenopus microsurgical manipulations, embryo mounts and scoring of kidney

711 phenotypes in NF stage 40 embryos. Zeiss LSM800 microscope with Airyscan detector,

712 LeicaSP5 and NikonA1 were used for confocal imaging of fixed and live samples. Captured

713 images and time-lapse movies were exported as original files and processed using ImageJ (Fiji

714 plugin). Image panels were built using FigureJ plugin. Final figures were assembled in Adobe

715 Photoshop CC. TEM imaging was carried out in a JEM 1010 transmission electron microscope

716 (JEOL, USA, Inc., Peabody, MA) with the AMT Imaging System (Advanced Microscopy

717 Techniques Corp, Danvers, MA).

719 Quantification and statistical analyses

720 ImageJ (Fiji plugin) software was used for quantitative image analyses. For quantification of E-

721 cadherin and F-actin staining (Figures 3, 5 and 6) images were captured using the same 
722 settings. The mean fluorescence intensity along the length of selected junction was measured.

723 To analyze tissue organization during nephron development (Figure 3) cells were manually

724 counted using the Cell Counter tool. The shortest distance between two neighboring nuclei was

725 measured using the straight-line selection tool. The area and circularity were measured using

726 the Analyze Particles tool. Wound area (Figure 7) was also measured using the Analyze

727 Particles tool. To obtain traveled distance, trajectories of randomly selected cells were traced

728 manually in each frame of the time-lapse using Plugin for Motion Tracking and Analysis

729 (MTrackJ) (The Biomedical Imaging Group of Erasmus University Medical Centre, Rotterdam,

730 The Netherlands). All experiments were repeated at least two times, with the exception of TEM

731 studies, which due to the prohibitive costs of the experiment represent single-trial analyses. The

732 exact sample size and statistical analysis for each experiment are presented in the

733 corresponding figure legend. Statistical analyses were carried out using GraphPad Prism 8.0

734 software.

735

736

737

738

739

740

741

742

743

744

745

746

747

748

749

750

751

752

753

754

755

756

\section{REFERENCES}

Adams, C.L., Chen, Y.T., Smith, S.J., and Nelson, W.J. (1998). Mechanisms of epithelial cellcell adhesion and cell compaction revealed by high-resolution tracking of E-cadherin-green fluorescent protein. J. Cell Biol. 142, 1105-1119.

Ang, S.F., Zhao, Z.S., Lim, L., and Manser, E. (2010). DAAM1 is a formin required for centrosome re-orientation during cell migration. PLoS One 5, e13064.

Becker, B.E., and Gard, D.L. (2006). Visualization of the Cytoskeleton in Xenopus Oocytes and Eggs by Confocal Immunofluorescence Microscopy. In Methods Mol Biol., pp. 69-86.

Burkel, B.M., von Dassow, G., and Bement, W.M. (2007). Versatile fluorescent probes for actin filaments based on the actin-binding domain of utrophin. Cell Motil. Cytoskeleton 64, 822-832.

Cai, D., Chen, S.-C., Prasad, M., He, L., Wang, X., Choesmel-Cadamuro, V., Sawyer, J.K., Danuser, G., and Montell, D.J. (2014). Mechanical feedback through E-cadherin promotes direction sensing during collective cell migration. Cell 157, 1146-1159.

Campbell, K., and Casanova, J. (2015). A role for E-cadherin in ensuring cohesive migration of a heterogeneous population of non-epithelial cells. Nat. Commun. 6, 7998.

Campbell, K., and Casanova, J. (2016). A common framework for EMT and collective cell migration. Dev. 143, 4291-4300.

Capaldo, C.T., and Macara, I.G. (2007). Depletion of E-Cadherin Disrupts Establishment but 
Not Maintenance of Cell Junctions in Madin-Darby Canine Kidney Epithelial Cells. Mol. Biol. Cell 18, 189-200.

Castelli, M., Boca, M., Chiaravalli, M., Ramalingam, H., Rowe, I., Distefano, G., Carroll, T., and Boletta, A. (2013). Polycystin-1 binds Par3/aPKC and controls convergent extension during renal tubular morphogenesis. Nat. Commun. 4, 1-10.

Cavey, M., Rauzi, M., Lenne, P.F., and Lecuit, T. (2008). A two-tiered mechanism for stabilization and immobilization of E-cadherin. Nature 453, 751-756.

Cohen, D.J., Gloerich, M., and Nelson, W.J. (2016). Epithelial self-healing is recapitulated by a 3D biomimetic E-cadherin junction. Proc. Natl. Acad. Sci. U. S. A. 113, 14698-14703. Collins, C., Denisin, A.K., Pruitt, B.L., and Nelson, W.J. (2017). Changes in E-cadherin rigidity sensing regulate cell adhesion. Proc. Natl. Acad. Sci. U. S. A. 114, E5835-E5844.

Combes, A.N., Davies, J.A., and Little, M.H. (2015). Cell-cell interactions driving kidney morphogenesis. Curr. Top. Dev. Biol. 112, 467-508. Corkins, M.E., Hanania, H.L., Krneta-Stankic, V., Delay, B.D., Pearl, E.J., Lee, M., Ji, H., Davidson, A.J., Horb, M.E., and Miller, R.K. (2018). Transgenic Xenopus laevis line for in vivo labeling of nephrons within the kidney. Genes (Basel). 9, 197.

Corkins, M.E., Krneta-Stankic, V., Kloc, M., McCrea, P.D., Gladden, A.B., and Miller, R.K. (2019). Divergent roles of the Wnt/PCP Formin Daam1 in renal ciliogenesis. PLoS One 14, e0221698.

Davidson, L.A., Marsden, M., Keller, R., and DeSimone, D.W. (2006). Integrin a5ß1 and Fibronectin Regulate Polarized Cell Protrusions Required for Xenopus Convergence and Extension. Curr. Biol. 16, 833-844.

DeLay, B.D., Krneta-Stankic, V., and Miller, R.K. (2016). Technique to Target Microinjection to the Developing Xenopus Kidney. J. Vis. Exp. 111.

DeLay, B.D., Corkins, M.E., Hanania, H.L., Salanga, M., Deng, J.M., Sudou, N., Taira, M., Horb, M.E., and Miller, R.K. (2018). Tissue-Specific Gene Inactivation in Xenopus laevis: Knockout of Ihx1 in the Kidney with CRISPR/Cas9. Genetics 208, 673-686.

\section{Friedl, P., and Mayor, R. (2017). Tuning Collective Cell Migration by Cell-Cell Junction}

Regulation. Cold Spring Harb. Perspect. Biol. 1-17.

Grikscheit, K., and Grosse, R. (2016). Formins at the Junction. Trends Biochem. Sci. 41, 148159.

Habas, R., Kato, Y., and He, X. (2001). Wnt/Frizzled activation of Rho regulates vertebrate gastrulation and requires a novel Formin homology protein Daam1. Cell 107, 843-854.

Hemmati-Brivanlou, A., and Melton, D.A. (1994). Inhibition of activin receptor signaling promotes neuralization in Xenopus. Cell 77, 273-281.

Higashi, T., Stephenson, R.E., and Miller, A.L. (2019). Comprehensive analysis of formin localization in Xenopus epithelial cells. Mol. Biol. Cell 30, 82-95.

Huebner, R.J., and Wallingford, J.B. (2018). Coming to Consensus: A Unifying Model Emerges for Convergent Extension. Dev. Cell 46, 389-396.

Huebner, R.J., Malmi-kakkada, A.N., Sarikaya, S., Weng, S., and Wallingford, J.B. (2020). Cadherin clustering controls heterogeneous, asymmetric junction dynamics during vertebrate axis elongation. BioRxiv 2020.02.11.944033.

800

Indra, I., Choi, J., Chen, C.S., Troyanovsky, R.B., Shapiro, L., Honig, B., and Troyanovsky, S.M. (2018). Spatial and temporal organization of cadherin in punctate adherens junctions. Proc. 
Natl. Acad. Sci. U. S. A. 115, E4406-E4415.

Jaiswal, R., Breitsprecher, D., Collins, A., Corrêa, I.R., Xu, M.Q., and Goode, B.L. (2013). The formin daam1 and fascin directly collaborate to promote filopodia formation. Curr. Biol. 23, 1373-1379.

Kamei, C.N., Gallegos, T.F., Liu, Y., Hukriede, N., and Drummond, I.A. (2019). Wnt signaling mediates new nephron formation during zebrafish kidney regeneration. Dev. 146.

Karner, C.M., Chirumamilla, R., Aoki, S., Igarashi, P., Wallingford, J.B., and Carroll, T.J. (2009). Wnt9b signaling regulates planar cell polarity and kidney tubule morphogenesis. Nat. Genet. 41, 793-799.

Kawabata Galbraith, K., Fujishima, K., Mizuno, H., Lee, S.J., Uemura, T., Sakimura, K., Formin DAAM1 in Dendritic Filopodia Determines Final Dendritic Configuration of Purkinje Cells. Cell Rep. 24, 95-106.e9.

Kida, Y.S., Sato, T., Miyasaka, K.Y., Suto, A., and Ogura, T. (2007). Daam1 regulates the endocytosis of EphB during the convergent extension of the zebrafish notochord. Proc. Natl. Acad. Sci. U. S. A. 104, 6708-6713.

Kim, H.Y., and Davidson, L.A. (2013). Microsurgical Approaches to Isolate Tissues from Xenopus Embryos for Imaging Morphogenesis. Cold Spring Harb Protoc 362-365.

Kim, S.W., Fang, X., Ji, H., Paulson, A.F., Daniel, J.M., Ciesiolka, M., van Roy, F., and McCrea, P.D. (2002). Isolation and Characterization of XKaiso, a Transcriptional Repressor That Associates with the Catenin Xp120 ctn in Xenopus laevis. J. Biol. Chem. 277, 8202-8208.

822 Krneta-Stankic, V., Sabillo, A., and Domingo, C.R. (2010). Temporal and spatial patterning of 823 axial myotome fibers in Xenopus laevis. Dev. Dyn. 239, 1162-1177.

824 Kunimoto, K., Bayly, R.D., Vladar, E.K., Vonderfecht, T., Gallagher, A.R., and Axelrod, J.D. (2017). Disruption of Core Planar Cell Polarity Signaling Regulates Renal Tubule Morphogenesis but Is Not Cystogenic. Curr. Biol. 27, 3120-3131.e4.

Lefevre, J.G., Chiu, H.S., Combes, A.N., Vanslambrouck, J.M., Ju, A., Hamilton, N.A., and Little, M.H. (2017). Self-organisation after embryonic kidney dissociation is driven via selective adhesion of ureteric epithelial cells. Dev. 144, 1087-1096.

Li, D., Hallett, M. a, Zhu, W., Rubart, M., Liu, Y., Yang, Z., Chen, H., Haneline, L.S., Chan, R.J., required for heart morphogenesis. Development 138, 303-315.

Li, J.X.H., Tang, V.W., and Brieher, W.M. (2020). Actin protrusions push at apical junctions to maintain E-cadherin adhesion. Proc. Natl. Acad. Sci. U. S. A. 117, 432-438. Walz, G. (2012). Vertebrate kidney tubules elongate using a planar cell polarity-dependent, rosette-based mechanism of convergent extension. Nat. Genet. 44, 1382-1387.

838 Liu, W., Sato, A., Khadka, D., Bharti, R., Diaz, H., Runnels, L.W., and Habas, R. (2008).

839 Mechanism of activation of the Formin protein Daam1. Proc. Natl. Acad. Sci. U. S. A. 105, 210840215.

841 Lu, J., Meng, W., Poy, F., Maiti, S., Goode, B.L., and Eck, M.J. (2007). Structure of the FH2 842 Domain of Daam1: Implications for Formin Regulation of Actin Assembly. J. Mol. Biol. 369, 843 1258-1269.

844 Marciano, D.K., Brakeman, P.R., Lee, C., Spivak, N., Eastburn, D.J., Bryant, D.M., lii, G.M.B., 
845

Hofmann, I., Mostov, K.E., Reichardt, L.F., et al. (2011). P120 catenin is required for normal renal tubulogenesis and glomerulogenesis. Development 138, 2099-2109. McMahon, A.P. (2016). Development of the Mammalian Kidney. Curr. Top. Dev. Biol. 31-64. Mendonsa, A.M., Na, T.Y., and Gumbiner, B.M. (2018). E-cadherin in contact inhibition and cancer. Oncogene 37, 4769-4780.

Miller, R.K., and McCrea, P.D. (2009). Wnt to build a tube: Contributions of Wnt signaling to epithelial tubulogenesis. Dev. Dyn. 239, NA-NA.

Miller, R.K., Gomez de la Torre Canny, S., Jang, C.-W., Cho, K., Ji, H., Wagner, D.S., Jones, E.A., Habas, R., and McCrea, P.D. (2011). Pronephric Tubulogenesis Requires Daam1Mediated Planar Cell Polarity Signaling. J. Am. Soc. Nephrol. 22, 1654-1664.

Moody, S.A., and Kline, M.J. (1990). Segregation of fate during cleavage of frog (Xenopus laevis) blastomeres. Anat. Embryol. (Berl). 182, 347-362.

Nagy, I.I., Xu, Q., Naillat, F., Ali, N., Miinalainen, I., Samoylenko, A., and Vainio, S.J. (2016). Impairment of Wnt11 function leads to kidney tubular abnormalities and secondary glomerular cystogenesis. BMC Dev. Biol. 16, 1-14.

Nieuwkoop, P.D., and Faber, J. (1994). Normal table of Xenopus laevis (Daudin) : a systematical and chronological survey of the development from the fertilized egg till the end of metamorphosis (New York: Garland Publishing, Inc.).

Nishimura, T., Honda, H., and Takeichi, M. (2012). Planar cell polarity links axes of spatial dynamics in neural-tube closure. Cell 149, 1084-1097.

Nishimura, T., Ito, S., Saito, H., Hiver, S., Shigetomi, K., Ikenouchi, J., and Takeichi, M. (2016). DAAM1 stabilizes epithelial junctions by restraining WAVE complex-dependent lateral membrane motility. J. Cell Biol. 215, 559-573.

Nworu, C.U., Krieg, P.A., and Gregorio, C.C. (2014). Preparation of developing Xenopus muscle for sarcomeric protein localization by high-resolution imaging. Methods 66, 370-379.

O'Brien, L.L., Combes, A.N., Short, K.M., Lindström, N.O., Whitney, P.H., Cullen-McEwen, L.A., Ju, A., Abdelhalim, A., Michos, O., Bertram, J.F., et al. (2018). Wnt11 directs nephron progenitor polarity and motile behavior ultimately determining nephron endowment. Elife 7, 1-25.

\section{Saxen, L. (1987). Organogenesis of the Kidney (Cambridge University Press).}

Shindo, A., and Wallingford, J.B. (2014). PCP and septins compartmentalize cortical actomyosin to direct collective cell movement. Science 343, 649-652.

Sive, H.L., Grainger, R.M., and Harland, R.M. (2000). Early development of Xenopus laevis : a laboratory manual (Cold Spring Harbor Press, Cold Spring Harbor, NY, USA: Cold Spring Harbor Laboratory Press).

Strickland, L., Von Dassow, G., Ellenberg, J., Foe, V., Lenart, P., and Burgess, D. (2004). Light microscopy of echinoderm embryos. Methods Cell Biol. 2004, 371-409.

Tada, M., and Heisenberg, C.P. (2012). Convergent extension: using collective cell migration and cell intercalation to shape embryos. Development 139, 3897-3904.

Takeichi, M. (2014). Dynamic contacts: Rearranging adherens junctions to drive epithelial remodelling. Nat. Rev. Mol. Cell Biol. 15, 397-410.

Venegas-FERRíN, M., Sudou, N., Taira, M., and Del Pino, E.M. (2010). Comparison of Lim1 expression in embryos of frogs with different modes of reproduction. Int. J. Dev. Biol. 54, 195202. 
888 Vestweber, D., Kemler, R., and Ekblom, P. (1985). Cell-adhesion molecule uvomorulin during 889 kidney development. Dev. Biol. 112, 213-221.

890 Vize, P.D., Carroll, T.J., and Wallingford, J.B. (2003). Induction, Development, and Physiology 891 of the Pronephric Tubules. In The Kidney, P.D. Vize, A.S. Woolf, and J.B.L. Bard, eds. (San 892 Diego: Elsevier), pp. 19-50.

893 Williams, E., Villar-Prados, A., Bowser, J., Broaddus, R., and Gladden, A.B. (2017). Loss of 894 polarity alters proliferation and differentiation in low-grade endometrial cancers by disrupting 895 Notch signaling. PLoS One 12, e0189081.

896 Yamashita, M., Higashi, T., Suetsugu, S., Sato, Y., Ikeda, T., Shirakawa, R., Kita, T., Takenawa, 897 T., Horiuchi, H., Fukai, S., et al. (2007). Crystal structure of human DAAM1 formin homology 2 898 domain. Genes Cells 12, 1255-1265.

899 Yap, A.S., Gomez, G.A., and Parton, R.G. (2015). Adherens Junctions Revisualized: Organizing 900 Cadherins as Nanoassemblies. Dev. Cell 35, 12-20. 

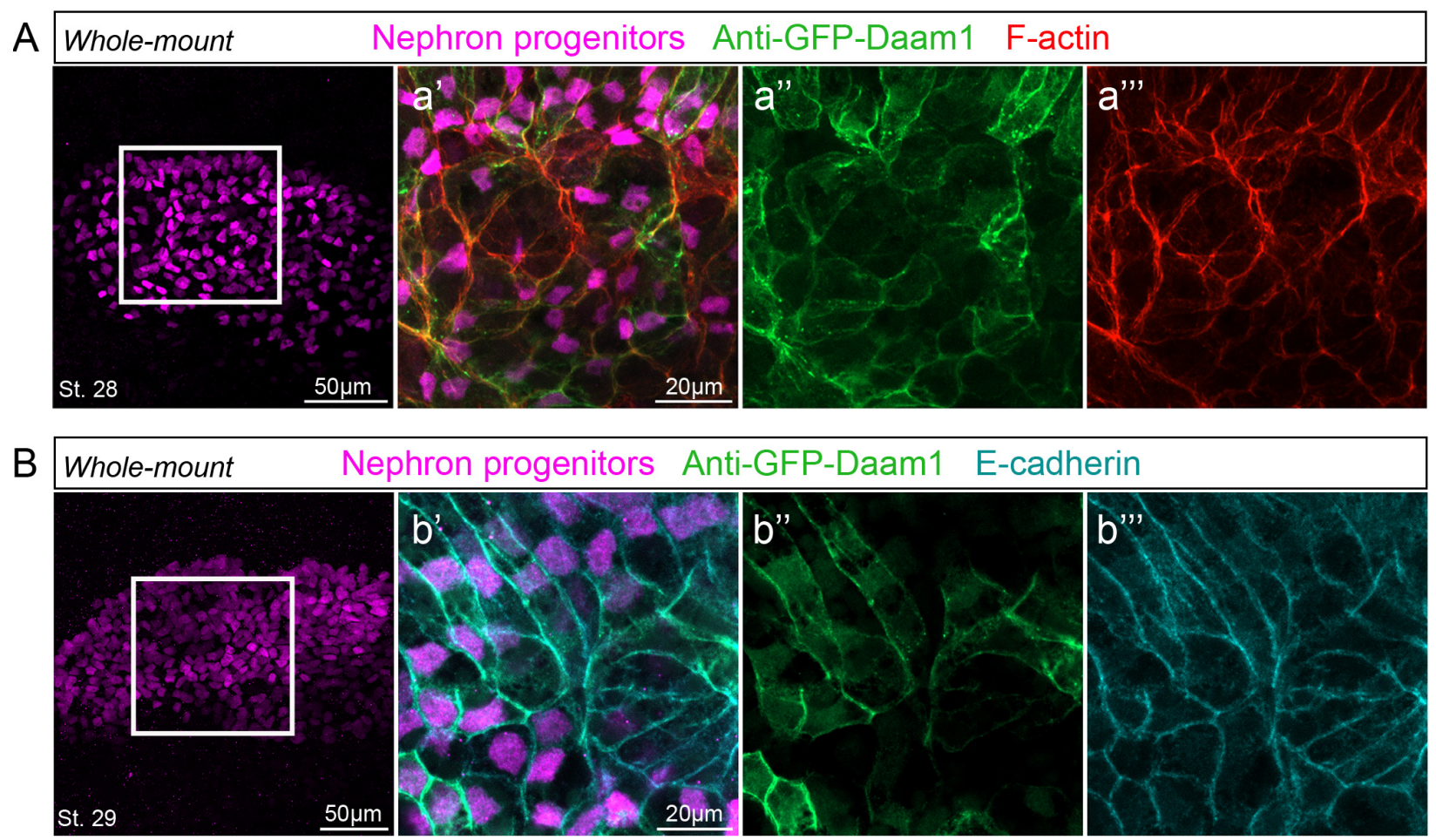

Figure 1. Daam1 co-localizes with junctional F-actin and E-cadherin during early nephron development Confocal maximum image projections of whole-mount immunostaining of Xenopus nephric primordium labeled by Lhx1(magenta) and GFP to visualize Daam1 (green) in conjunction with, (A) Phalloidin staining to visualize F-actin (red) or (B) E-cadherin (cyan). a'-a"' and b'-b"' represent close-up images of white boxes. 
A

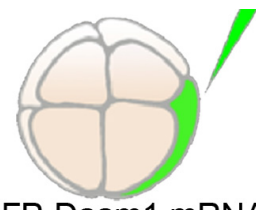

GFP-Daam1 mRNA

kidney-targeted

microinjections
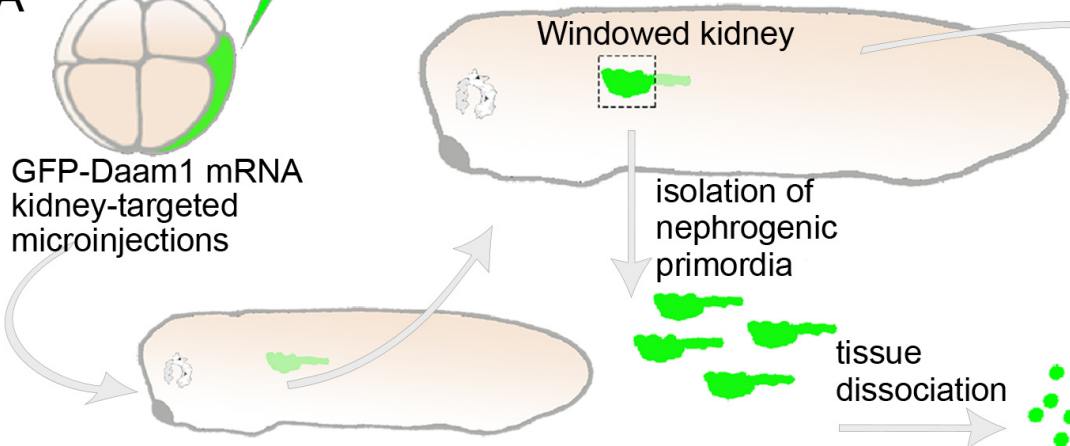

primordia
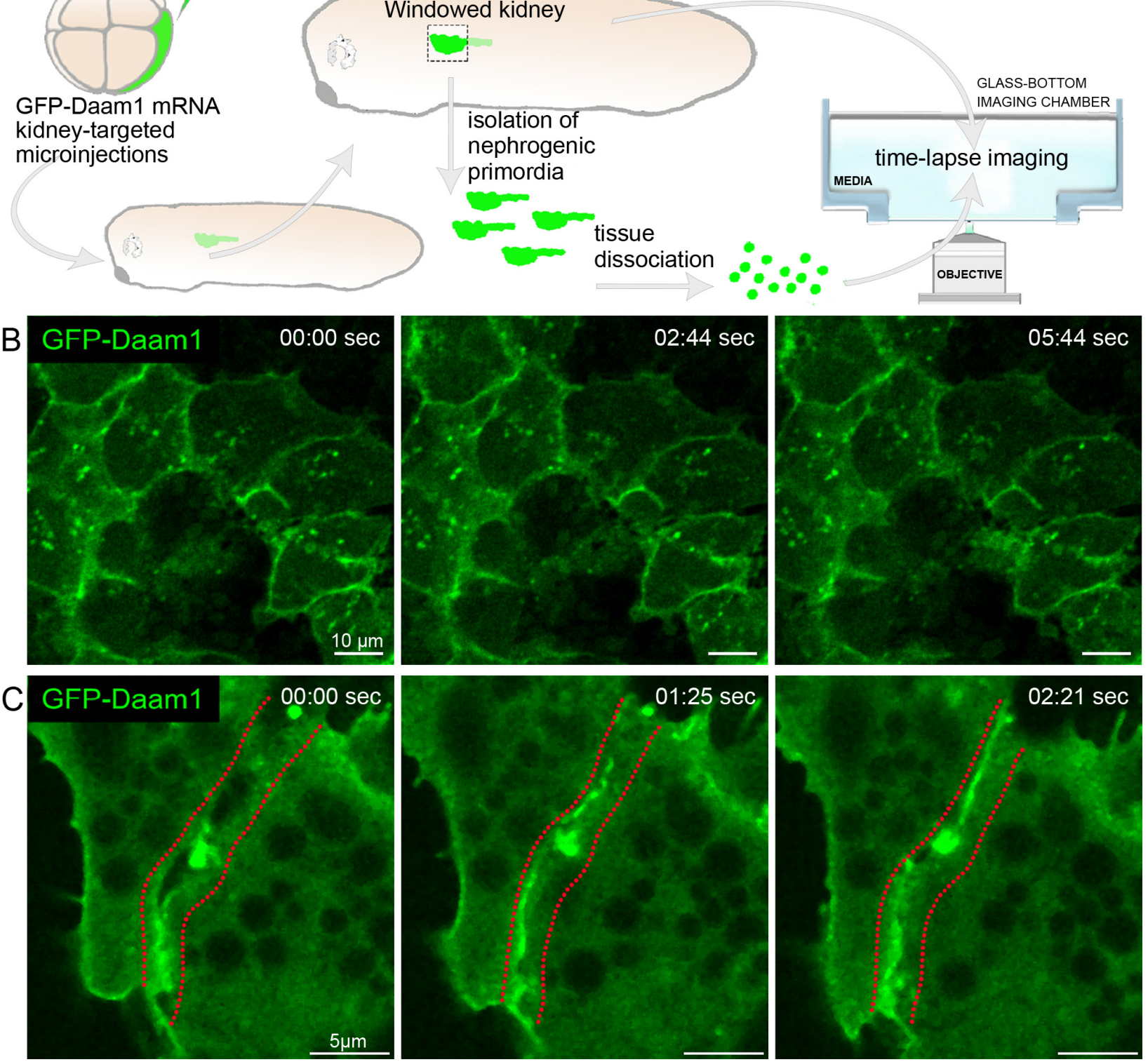

Figure 2. Daam1 localizes to newly formed cell-cell contacts

(A) Schematic illustration showing steps involved in preparation of "windowed kidney" embryos and primary cultures expressing GFP-Daam1. Please note that for clarity of illustration the 8-cell GFP-Daam1 injected Xenopus blastomere is fate-mapped strictly to the nephric primordium and that blastomere also contributes to epidermis, ventral and dorsal somites, hidgut, proctodeum and trunk neural crest cells (DeLay et al., 2016; Moody and Kline, 1990).

(B) Time-lapse imaging montage of the nephric primordium expressing GFP-Daam1 in "windowed kidney" embryos. Elapsed time is indicated at the top in seconds; see Video S1.

(C) Time-lapse imaging montage shows cells isolated from a developing nephron expressing GFP-Daam1 mRNA adhering with each other. Elapsed time is indicated at the top in seconds. The border arising between two cells is delineated by the red dotted line; see Video S2. 


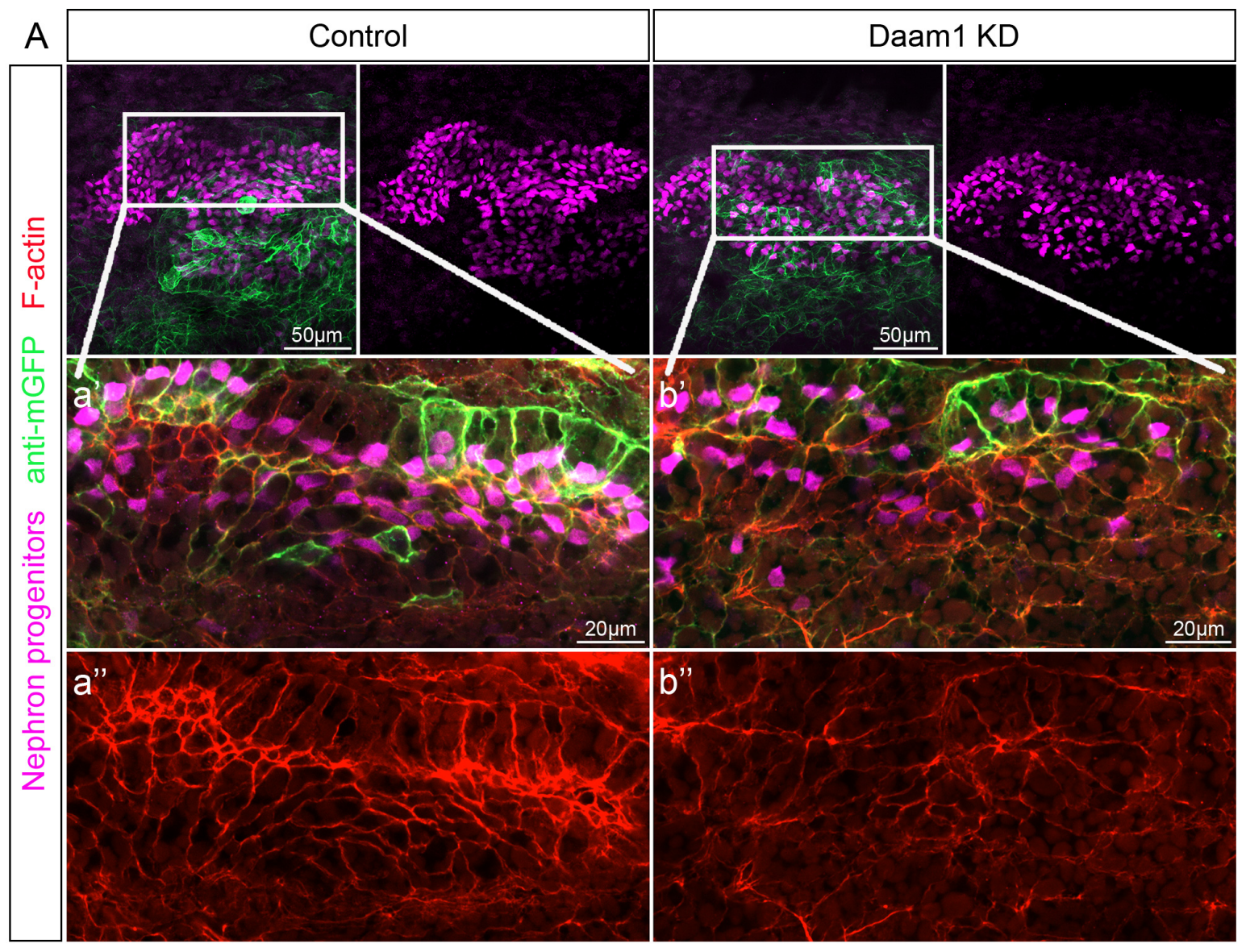

B

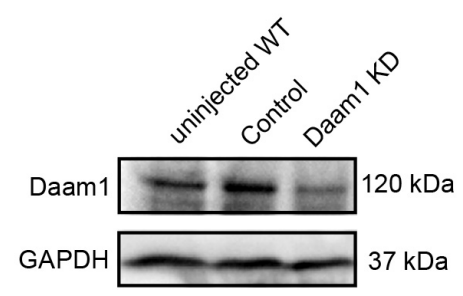

C

F

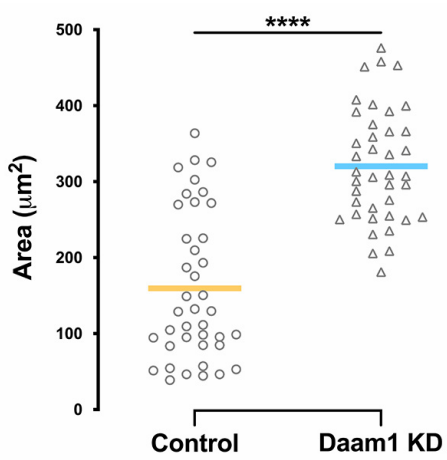

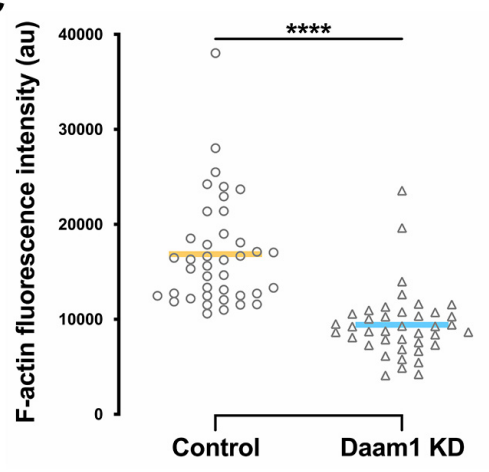

$D_{\underline{\underline{\underline{e}}}}$

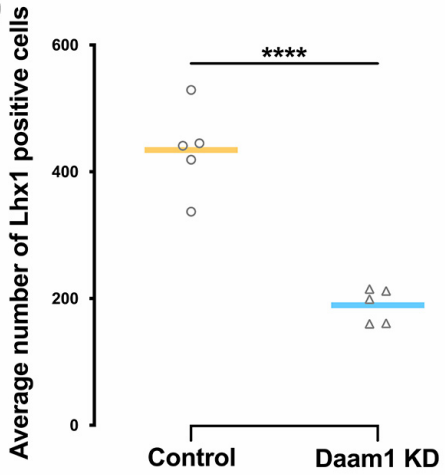

E
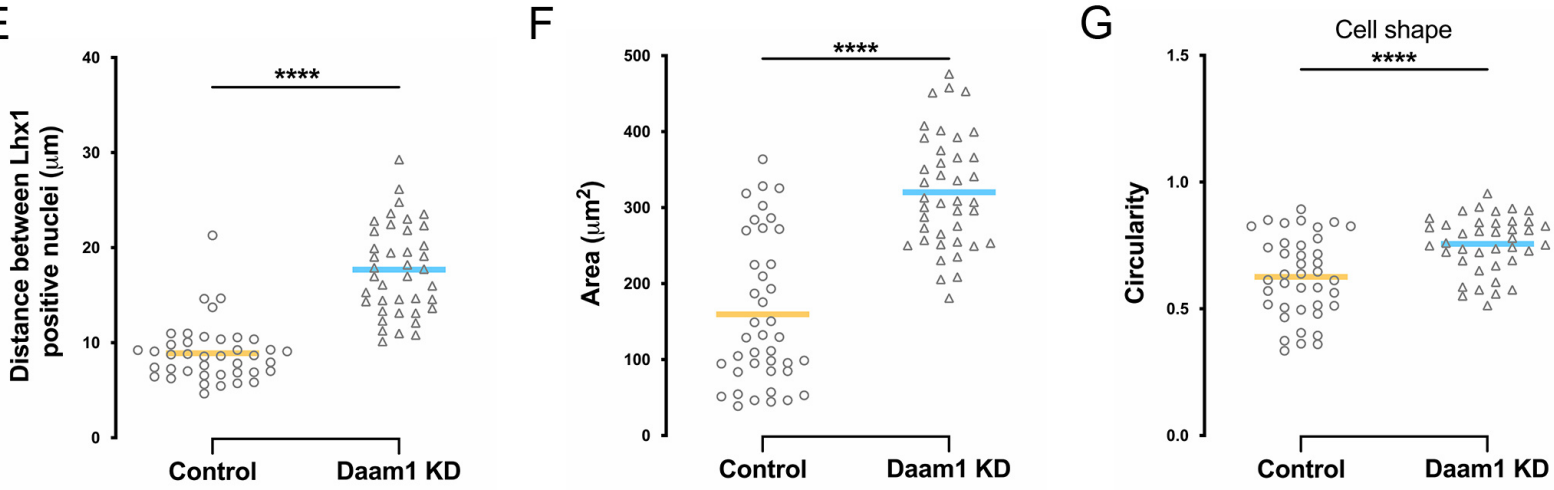
bioRxiv preprint doi: https://doi.org/10.1101/2020.08.18.256123; this version posted August 19, 2020. The copyright holder for this preprint (which was not certified by peer review) is the author/funder. All rights reserved. No reuse allowed without permission.

Figure 3. Effects of Daam1 depletion on the nephrogenic primordium (A) Maximum projection confocal images of F-actin expression (red) in nephric primordium (magenta) in Control and Daam1 knockdown embryos. a-a" and b'-b" represent close-up images of the corresponding regions in white boxes; see Videos S3 and S4.

(B) Western blot showing Daam1 and GAPDH (control) protein levels for uninjected wild type (WT) and Control (Standard morpholino) and Daam1 KD (Daam1 morpholino) injected embryos.

(C) The graph showing the relative fluorescence intensity levels of junctional F-actin in the nephric primordia of Control and Daam $1 \mathrm{KD}$ embryos. $\mathrm{N}_{\text {Control }}=40$ junctions on 2 embryos and $\mathrm{N}_{\text {Daam } 1 \mathrm{KD}}=40$ junctions on 2 embryos. ${ }^{* * *} \mathrm{P}<0.0001$ analyzed by unpaired t-test.

(D-G) Morphometric analyses of Control and Daam1-depleted nephric primordia. The thick bars represent the mean, ${ }^{* * * *} \mathrm{P}<0.0001$ analyzed by unpaired t-test, $(\mathrm{E}-\mathrm{G}) \mathrm{N}_{\text {Control }}=40$ junctions on 2 embryos and $\mathrm{N}_{\text {Daam } 1 \mathrm{KD}}=40$ junctions on 2 embryos. Graphs showing comparison between Control and Daam1-depleted nephric primordia of,

(D) the average number of Lhx1-positive nephron progenitors where $\mathrm{N}_{\text {Control }}=5$ embryos and $\mathrm{N}_{\text {Daam } 1 \mathrm{KD}}=5$ embryos,

(E) the relative distance between nearest neighbors of Lhx1-positive nuclei,

$(F)$ the relative cell area and

(G) the relative circularity, where 1 represents the perfect circle. 


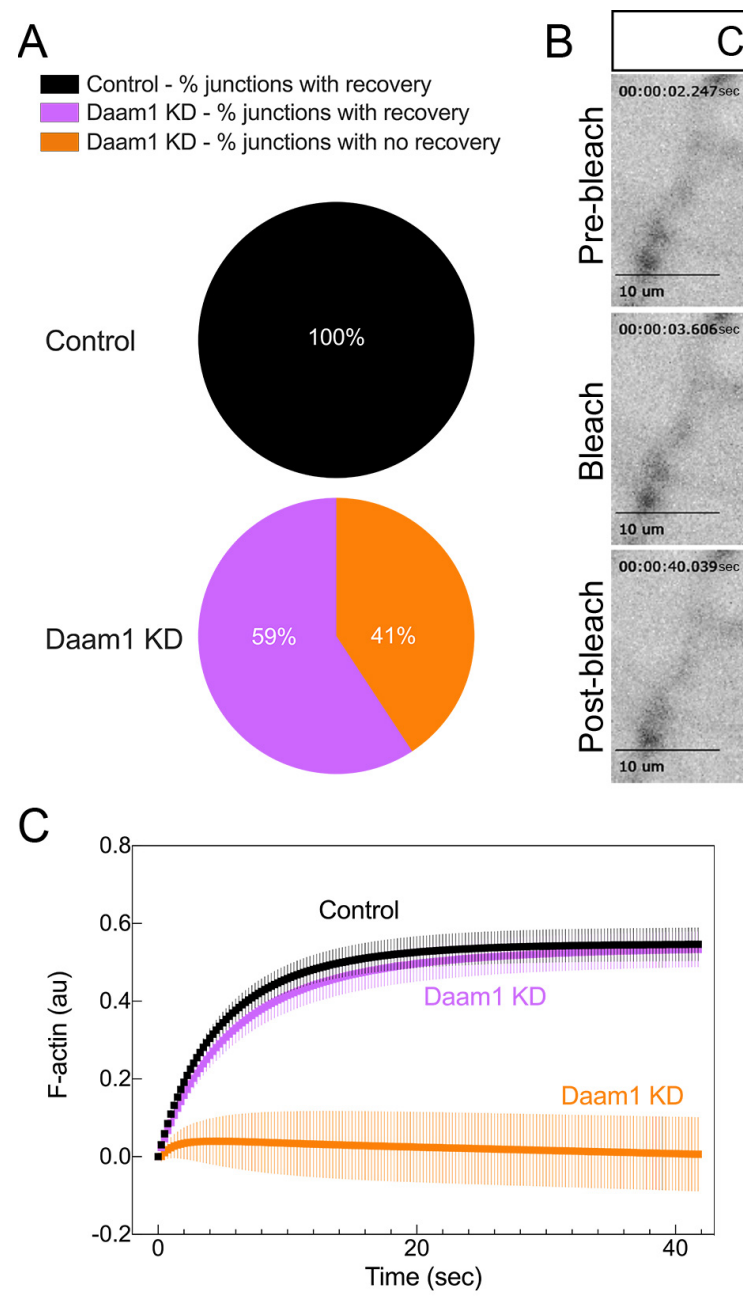

Figure 4. Daam1 regulates assembly of junctional F-actin in developing nephron F-actin dynamics at cell-cell junctions of Control and Daam1-depleted developing nephrons expressing mCherryUtrophin were assessed using FRAP.

(A) Percentage of junctions showing recovery of fluorescence after bleaching in Control (black, $\mathrm{N}_{\text {total }}=27$ junctions, $1-5$ junctions/embryo) and Daam1 KD (purple and orange, $\mathrm{N}_{\text {total }}=27$ junctions, 1-5 junctions/embryo) nephrons.

(B) Typical time-lapse images of Control and Daam1-depleted cell junctions before and after photobleaching. In each image, the bleached region is highlighted with a circle (black - Control junction showing recovery, purple - Daam1 KD junction showing recovery and orange - Daam1 KD junction showing no-recovery of fluorescence after photobleaching). (C) Graph shows average recovery curves obtained from individual best-fit plots for Control (black), Daam1 KD junctions with (purple) and without (orange) recovery of fluorescence after photobleaching.

(D-E) Bar graphs comparing Control and Daam1 KD profiles calculated from individual best-fit curves for Control (black) and Daam1 KD junctions with recovery of fluorescence after photobleaching (purple). Data represent the mean \pm S.E. from three independent experiments. P-values were analyzed by unpaired t-test.

(D) Bar graph of the relative half-times for F-actin.

(E) Bar graph of the relative mobile fraction for F-actin. 

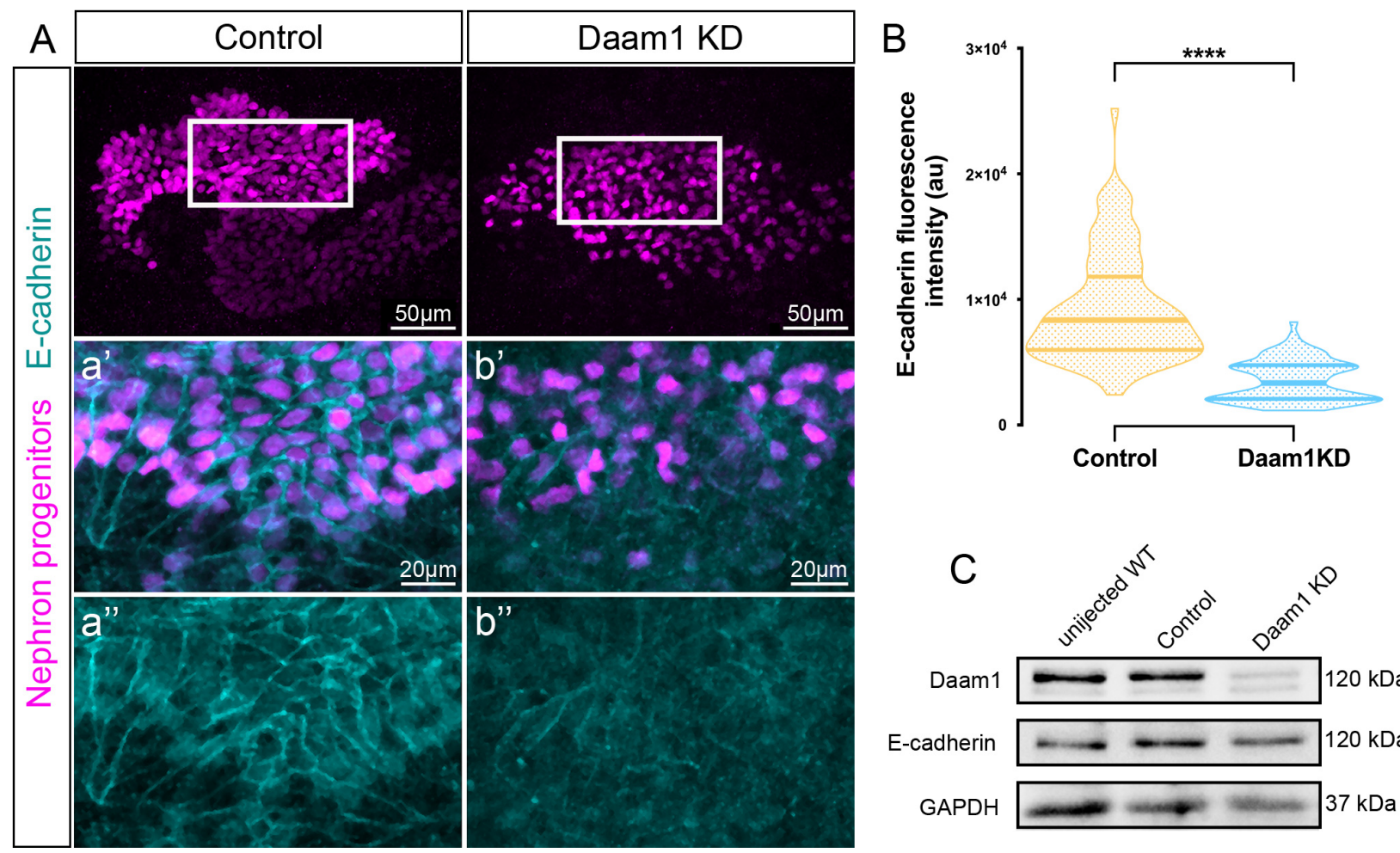

Figure 5. Daam1 promotes localization of junctional E-cadherin

(A) Maximum projection confocal images of E-cadherin expression (cyan) in nephric primordium (magenta) in Control and Daam1 KD embryos a-a' and b-b' represent the close-up images of corresponding regions in white boxes.

(B) Violin plots depicting the relative fluorescence intensity of junctional E-cadherin in the nephric primordia of Control (orange) and Daam1 KD (blue). $\mathrm{N}_{\text {Control }}=88$ junctions on 4 embryos and $\mathrm{N}_{\text {Daam } 1 \mathrm{KD}}=84$ junctions on 4 embryos. Centerlines represents median; Limits show $1^{\text {st }}$ and $3^{\text {rd }}$ quartile. ${ }^{* * *} \mathrm{P}<0.0001$ analyzed by unpaired t-test.

(C) Western blot showing Daam1, E-cadherin and GAPDH (control) protein levels in uninjected wild type (WT) and Control (Standard morpholino) and Daam1 KD (Daam1 morpholino) injected embryos. 

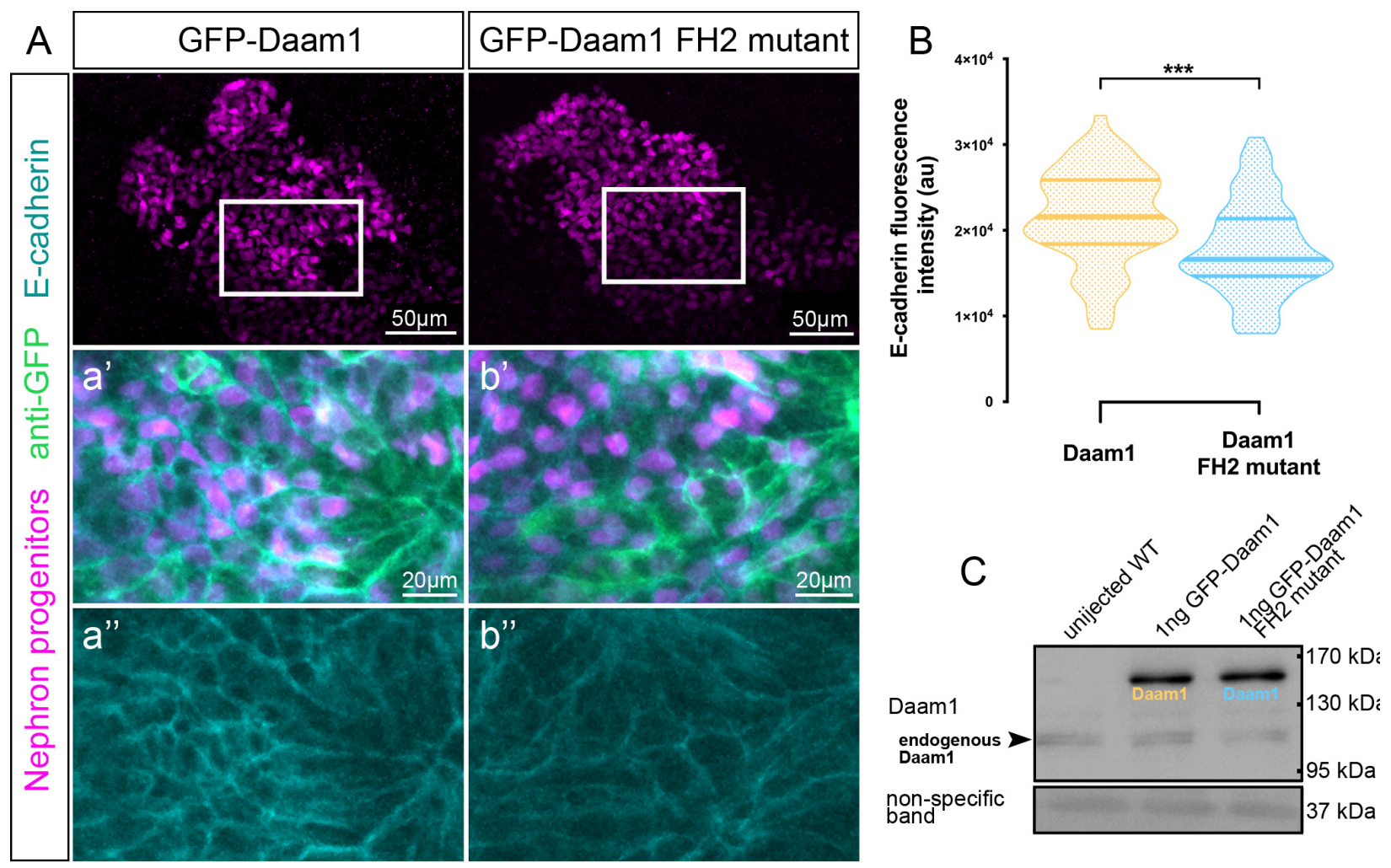

Figure 6. E-cadherin localization to cell-cell junctions is mediated by FH2 domain of Daam1

(A) Maximum projection confocal images showing E-cadherin staining (cyan) in nephric primordium (magenta) expressing GFP-Daam1 or GFP-Daam1 FH2 mutant mRNA. a-a' and b-b' represent close-up images of corresponding regions in white boxes.

(B) Violin plots depicting the relative fluorescence intensity of junctional E-cadherin in the nephric primordia expressing GFP-Daam1 (orange) and GFP-Daam1 FH2 mutant (blue) mRNA. N Daam1 $_{1}=60$ junctions on 3 embryos and $N_{\text {Daam1FH2mutant }}=55$ junctions on 3 embryos. Center-lines represents median; Limits show $1^{\text {st }}$ and $3^{\text {rd }}$ quartile. ${ }^{* * *} P<0.0002$ analyzed by unpaired t-test.

(C) Western blot showing the exogenous and endogenous protein levels of Daam1 in uninjected wild type (WT) embryos, embryos injected with $1 \mathrm{ng}$ of GFP-Daam1 mRNA and 1ng GFP-Daam1 FH2 mutant mRNA. The non-specific band confirms equal loading. 

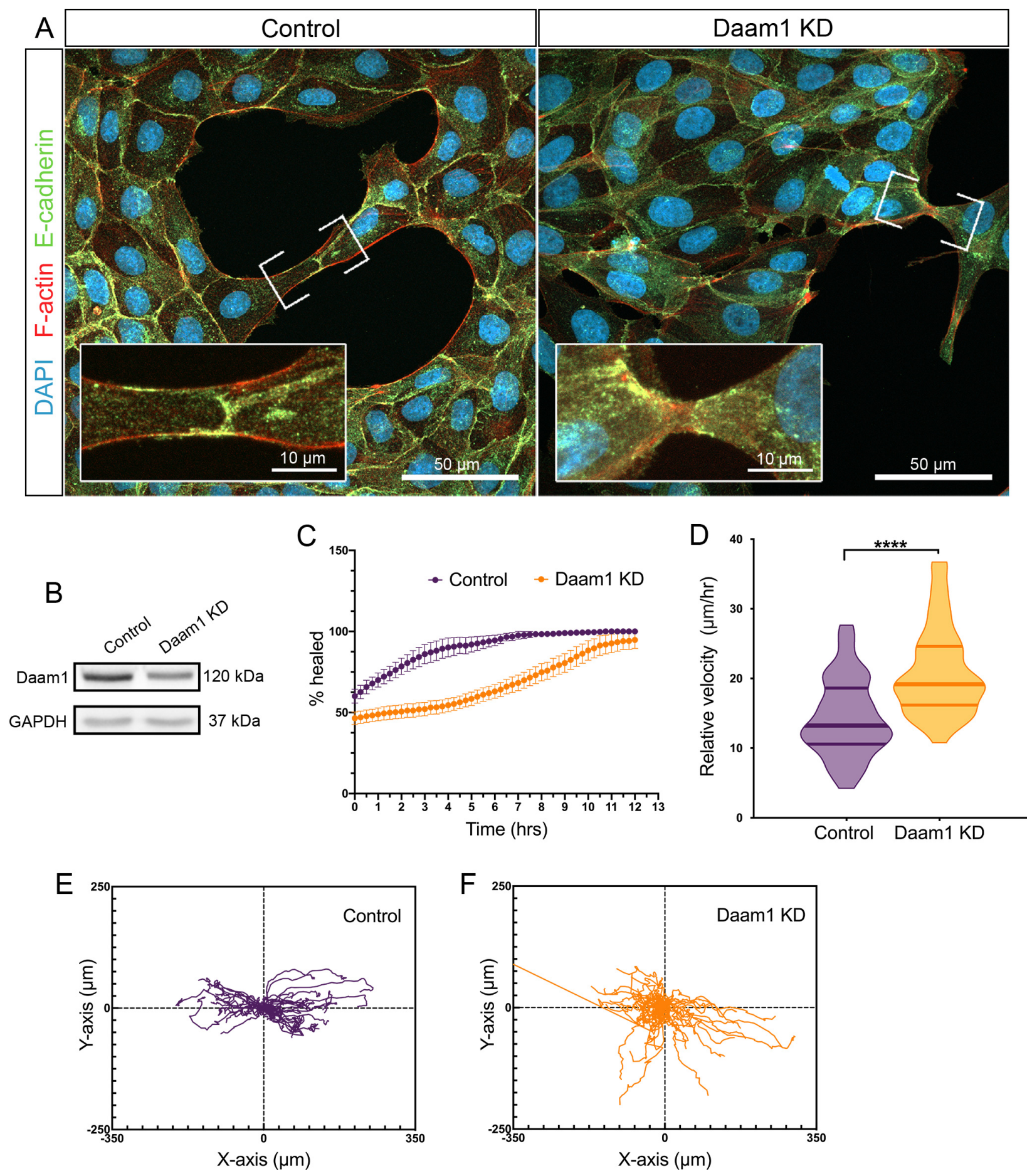
bioRxiv preprint doi: https://doi.org/10.1101/2020.08.18.256123; this version posted August 19, 2020. The copyright holder for this preprint (which was not certified by peer review) is the author/funder. All rights reserved. No reuse allowed without permission.

Figure 7. Daam1- depleted MDCK cells display compromised localization of E-cadherin at cell-cell contacts and impaired cohesion during collective movement

(A) E-cadherin (green), F-actin (red) and DAPI (blue) in subconfluent the MDCK Control and shDaam1 knockdown cells. E-cadherin localization in the nascent cell-cell contacts (marked by white brackets and shown enlarged in corresponding white boxes) is impaired in shDaam1- deficient cells.

(B) Western blot analysis of Daam1 and GAPDH protein levels in the MDCK Control and shDaam1 knockdown cells.

(C-F) Summary of the wound-healing experiments for the MDCK Control and Daam1 KD cells, see Video S7.

(C) Daam1 depletion impairs wound closure. The graph represents the percent of the wound surface area over time for Control (purple) and Daam1 KD (orange) cells. Error bars indicate S.E. of the mean on 4 assays.

(D-F) Manually tracking migration paths of single-cells during the wound closure demonstrates that Daam1 organizes collective movement of the MDCK epithelial monolayers by modulating the speed and directionality of individual cells. Depletion of Daam1 results in increased velocity and random migration. $\mathrm{N}_{\text {Control }}=52$ cells from 4 assays and $\mathrm{N}_{\text {Daam1 }}$ $\mathrm{KD}=42$ cells tracked from 4 assays. Cells were tracked in 15 minutes increments for 12 hours.

(D) Violin plots represent migration velocity calculated from tracking traveled distances of single cells for Control and Daam1 KD cells. Center-lines represents median; Limits show $1^{\text {st }}$ and $3^{\text {rd }}$ quartile. ${ }^{* * t} \mathrm{P}<0.0001$ analyzed by unpaired ttest.

(E) Wind-rose plot showing migration tracks of individual Control cells.

(F) Wind-rose plot showing migration tracks of Daam1 KD cells. 